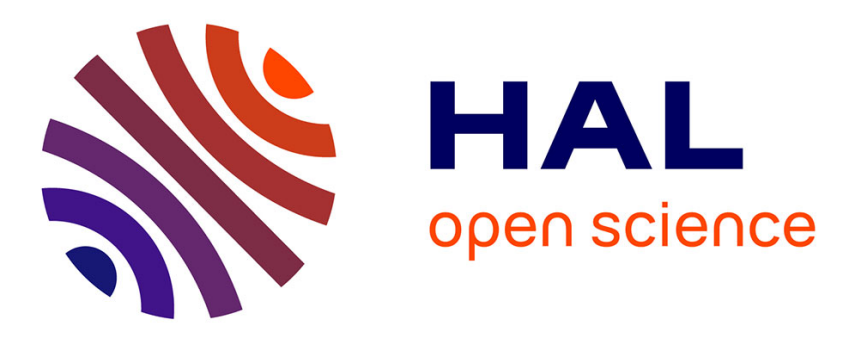

\title{
A First-Order Representation of Pure Type Systems Using Superdeduction
}

\author{
Guillaume Burel
}

\section{To cite this version:}

Guillaume Burel. A First-Order Representation of Pure Type Systems Using Superdeduction. 23rd Annual IEEE Symposium on Logic In Computer Science, Jun 2008, Pittsburgh, PA, United States. pp.253-263, 10.1109/LICS.2008.22 . inria-00198543

\section{HAL Id: inria-00198543 \\ https://inria.hal.science/inria-00198543}

Submitted on 17 Dec 2007

HAL is a multi-disciplinary open access archive for the deposit and dissemination of scientific research documents, whether they are published or not. The documents may come from teaching and research institutions in France or abroad, or from public or private research centers.
L'archive ouverte pluridisciplinaire $\mathbf{H A L}$, est destinée au dépôt et à la diffusion de documents scientifiques de niveau recherche, publiés ou non, émanant des établissements d'enseignement et de recherche français ou étrangers, des laboratoires publics ou privés. 


\title{
Superdeduction as a logical framework
}

\author{
Guillaume Burel \\ Nancy-Université (Université Henri Poincaré) \& LORIA (UMR 7503 CNRS-INPL-INRIA-Nancy2-UHP) \\ E-mail: guillaume.bureldens-lyon.org
}

\begin{abstract}
Superdeduction is a formalism inspired from deduction modulo which permits to enrich a first-order deduction system-such as natural deduction or sequent calculuswith new inference rules automatically computed from the presentation of a theory. We give a natural encoding from every Pure Type System (PTS) into superdeduction by defining an appropriate first-order theory. We prove that this translation is correct and conservative, showing a correspondence between valid typing judgment in the PTS and provable sequents in superdeduction.

As a byproduct, we also introduce the superdeductive sequent calculus for intuitionistic logic, which was until now only defined for classical logic. We show its equivalence with the superdeductive natural deduction. We also prove that for a large class of theories, the newly created rules suffice to shows all results related to the theory only.

These results leads to a better understanding of the implementation and the automation of proof search for PTS, as well as to more cooperation between proof assistants.
\end{abstract}

\section{Introduction}

According to Pfenning [20], a logical framework is a meta-language for the specification of deductive systems. The most famous one is the Edinburgh Logical Framework introduced in [17], which is based on a $\lambda$-calculus with dependent types which is known as $\lambda P$ or $\lambda \Pi$. Many systems, such as first-order natural deduction, simply typed $\lambda$ calculus, sundry presentations of modal logics, etc. can be represented in it.

Most existing interactive theorem provers are based on the logical framework approach, either explicitly as in Isabelle (http://isabelle.in.tum.de/) or implicitly in proof assistants based on extensions of $\lambda \Pi$, such as Coq (http://coq.inria.fr/). These extensions are instances of what are called Pure Type Systems (PTS), which are generic typing systems for $\lambda$-calculi with dependent types.

Superdeduction consists quite in the inverse approach to logical frameworks: from the presentation of a theory is computed a deductive system which is well adapted to search for proofs in this theory. For instance, from the intuitive definition of the inclusion $A \subseteq B \equiv \forall x . x \in A \Rightarrow x \in B$ are computed the rules

$\subseteq_{I}^{\mathrm{df}} \frac{\Gamma, x \in A \vdash x \in B}{\Gamma \vdash A \subseteq B} x_{\text {free in } \Gamma} \subseteq_{E}^{\mathrm{df}} \frac{\Gamma \vdash A \subseteq B \quad \Gamma \vdash t \in A}{\Gamma \vdash t \in B}$

in supernatural deduction modulo, the superdeductive system based on natural deduction.

The origins of superdeduction are to be found in deduction modulo [13]. Deduction modulo was originally thought of as a way to make computation and deduction easily cooperate in proofs. This is done by modeling the computations through a congruence between propositions, and by applying the inference rules of the deductive system we are considering modulo this congruence. For the instance of the inclusion above, this means that the following derivation is valid in natural deduction modulo:

$$
\forall_{I} \frac{\Gamma \vdash x \in A \Rightarrow x \in B}{\Gamma \vdash A \subseteq B} \quad \begin{aligned}
& x \text { not free in } \Gamma \\
& A \subseteq B \equiv \forall x . x \in A \Rightarrow x \in B
\end{aligned}
$$

We can remark that above this derivation it is quite natural to apply $\Rightarrow_{I}$. This explains why the new rule $\subseteq_{I}^{\mathrm{df}}$, where this step is precomputed, is more adapted to build proofs in the theory.

Notwithstanding, superdeduction and deduction modulo can be seen as a logical framework. In particular, HOL was presented in deduction modulo [12], and this is also possible in superdeduction, leading to new inference rules which correspond exactly to the ones of HOL- $\lambda$. This presentation is not only interesting for practical reasons, leading to automated theorem proving procedures based on deduction modulo, such as ENAR [13] or TaMed [6], and well adapted for HOL. It also permits to get a first-order characterization of HOL. Indeed, proving in the sequent calculus modulo, or in supernatural deduction, is equivalent to proving in the pure sequent calculus within a so called compatible (firstorder) theory (see [13, Proposition 1.8]).

An important issue in interactive theorem proving is the abundance of proof assistants. Ideally, we would like to 
be able to use theories developed in a particular theorem prover into another one, and thus to have translations between all the proof systems corresponding to the provers. Another approach was proposed by Kirchner through Fellowship [21]. This tool permits to translate proofs in the first-order sequent calculus into proofs in PVS (http:// pws.csl.sri.com/), Coq (http://coq.inria.fr/) and soon other provers. Recently, another step was performed by Cousineau and Dowek [9] who proved that every Pure Type System can be encoded in $\lambda \Pi$ modulo. Inversely to Fellowship, this permits to translate proofs of the theorem provers into proofs of $\lambda \Pi$ modulo, which can then be checked by the tool Europa.

The question that naturally arises is why this encoding is not performed in a first-order system (i.e. without $\beta$ conversion, which is a higher-order mechanism) such as for instance the sequent calculus modulo, as was done for HOL. This paper shows how it is possible. Then, using the equivalence between proving in deduction modulo or superdeduction and proving using a compatible theory, this will give us proofs in the first-order sequent calculus (without modulo), which can be fed to Fellowship. Furthermore, this leads to the automation of proof search in PTS, either through automated provers based on deduction modulo (TaMed and ENAR), or, using the equivalence, through standard automated provers.

To encode the PTS, we define a first-order theory which produces, in supernatural deduction, new inference rules that are close to the typing rules of the PTS. In particular, in a PTS, there is a conversion rule that say that two $\beta$ convertible terms type the same terms. To simulate this in a first-order setting, we will use some $\lambda$-calculus with explicit substitutions, such as for instance $\lambda_{\sigma}[1]$. As we do not want that our work depends on a particular calculus, we use a scheme of calculi, quite the same as Kesner [18] (who used it to get a generic proof of confluence of such calculi). We then prove that our translation is correct-valid typing judgments of the PTS can be translated into provable sequents in supernatural deduction - and conservative-provable sequents in supernatural deduction corresponds (modulo $\beta$ reduction) to valid typing judgments.

To prove the conservativeness, we need the fact that the newly created rules are enough to prove everything related to the first-order theory. To prove this, we define correspondences between supernatural deduction and the superdeductive system for the intuitionistic sequent calculus. The socalled super sequent calculus [8] being until now only introduced for classical logic, we define it here. Because we need as few permutation problems as possible for technical reasons, we do not base the intuitionistic super sequent calculus on Gentzen sequent calculus for intuitionistic logic LJ, but on the sequent calculus LB [22]. In addition to a better comprehension of the properties of the supernatural de-

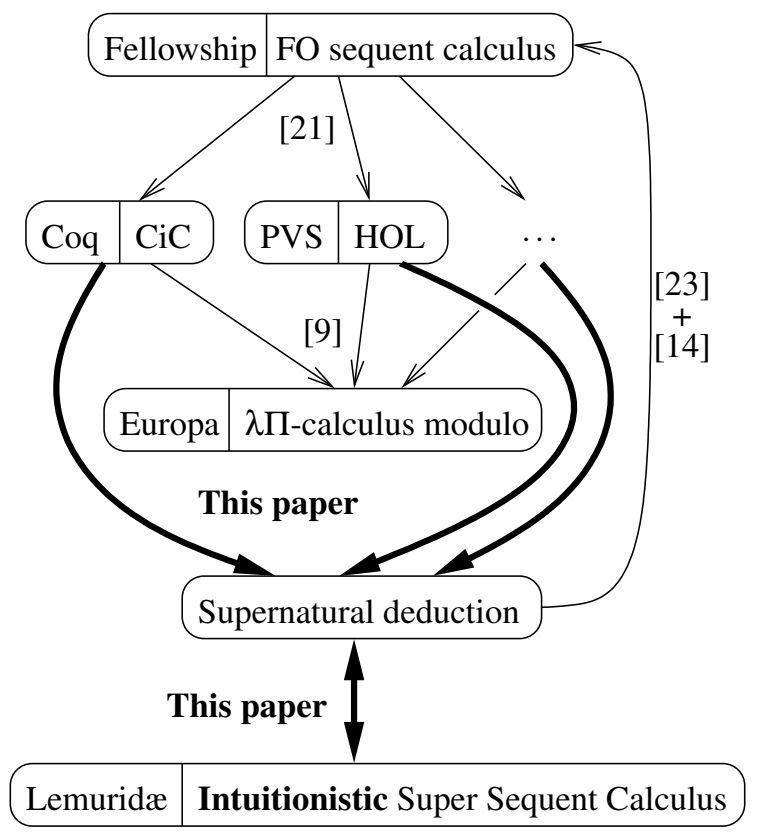

\section{Figure 1. Complementarity of our work with existing systems}

duction as a logical framework, this also leads to the actual use of its super sequent calculus counterpart in the theorem prover based on superdeduction, Lemuridæ [8], in a proven sound and correct way.

The contributions of this paper are summed up in figure 1. We show that every PTS can be encoded in a natural way in supernatural deduction through the definition of a first-order theory. We prove that this translation is correct and conservative. This shows that superdeduction is expressive enough to encode complicated deductive systems, and can therefore be considered as a powerful logical framework. We also prove an equivalence between supernatural deduction and super sequent calculus, and this implies that the new super rules are enough.

The next section presents the notions necessary to the remaining of the paper, i.e. LB, PTS, and the scheme of calculi with explicit substitutions. Section 3 introduces the two systems of superdeduction, i.e. supernatural deduction and super sequent calculus. The translation between is given, as well as a theorem (Corollary 10) stating that the new inference rules are enough to prove results related only to the theory used to compute the rules, for a large class of theories. Section 4 defines the first-order theory used to encode a PTS, and presents the supernatural deduction rules computed from it. Their soundness and conservativeness is then proved. Finally, section 5 presents some results following from this encoding. 


\section{Prerequisites}

The reader is assumed to be familiar with the natural deduction $\mathrm{NJ}$ and the sequent calculi $\mathrm{LJ}$ and $\mathrm{LK}$ of Gentzen [14], and to have basic notions on rewriting, as can be found in [3], which notations we will use. We restrict ourselves to intuitionistic first-order logic with only $\Rightarrow, \wedge$ as connectors and $\forall$ as quantifier. This is enough for the remaining of the paper, but this could be generalized to full intuitionistic first-order logic.

\subsection{Intuitionistic sequent calculus}

The standard sequent calculus for first-order intuitionistic logic is the one introduced by Gentzen [14]. It is the sequent calculus for first-order classical logic restricted to sequents with at most one formula in the right part. Unfortunately, this calculus permits too few permutations between inference rules, and therefore the super sequent calculus based on it would be incomplete even for simple theories. We will therefore use another sequent calculus, LB [22], which is based on a tableau method for intuitionistic logic and allows more permutations. The inference rules for LB are the following:

$$
\begin{array}{cc}
\text { Axiom } \frac{\text { Cut }}{\Gamma, A \vdash A, \Delta} & \wedge_{R} \frac{\Gamma \vdash A, \Delta \quad \Gamma \quad \Gamma \vdash A, \Delta}{\Gamma \vdash A \wedge B, \Delta} \\
\wedge_{L} \frac{\Gamma, A, B \vdash \Delta}{\Gamma, A \wedge B \vdash \Delta} & \quad \Rightarrow_{R} \frac{\Gamma, A \vdash B}{\Gamma \vdash A \Rightarrow B, \Delta} \\
\Rightarrow_{L} \frac{\Gamma, B \vdash \Delta \quad \Gamma \vdash A, \Delta}{\Gamma, A \Rightarrow B \vdash \Delta} & \quad \forall_{R} \frac{\Gamma \vdash A}{\Gamma \vdash \forall x . A, \Delta} x \text { not free in } \Gamma
\end{array}
$$

Note how, on $\Rightarrow_{R}$ and $\forall_{R}, \Delta$ is discarded in the above sequent. This is why this sequent calculus corresponds to intuitionistic logic. Axiom and Cut are called identity rules, the others logical rules.

\subsection{Pure Type Systems}

PTS are typing systems for the $\lambda$-calculus with dependent type. In particular, the simply-typed $\lambda$-calculus, $\lambda \Pi$, and the calculus of constructions (in fact, the whole $\lambda$-cube), $\lambda \mathrm{HOL}$, can be considered as PTS. We refer to [4]

Definition 1 (Pure Type Systems). A PTS is defined by three data: a set $\mathcal{S}$ of sorts, a relation $\mathcal{A} \subseteq \mathcal{S} \times \mathcal{S}$ of axioms, and a relation $\mathcal{R} \subseteq \mathcal{S} \times \mathcal{S} \times \mathcal{S}$ of rules.

Pseudo-terms are defined using this grammar $(s \in \mathcal{S})$ :

$$
T \stackrel{!}{=} x|s| \Pi x: T T|\lambda x: T T|(T T) .
$$

The typing rules are represented in figure 2. A term of the PTS is a pseudo-term which can be typed.
Any PTS has the following properties:

Lemma 1 (Subject reduction [4, Theorem 5.2.15]). If $\Gamma \vdash_{\mathrm{PTS}} A: B$ and $A \stackrel{*}{\longrightarrow} A^{\prime}$ then $\Gamma \vdash_{\mathrm{PTS}} A^{\prime}: B$.

Lemma 2 (Subject reduction for $\eta$ [15, Proposition 2.6]). If $\Gamma \vdash_{\mathrm{PTS}} \lambda x: A(T x): B$ then $\Gamma \vdash_{\mathrm{PTS}} T: B$.

Lemma 3 (Correctness of types [4, Corollary 5.2.14]). If $\Gamma \vdash_{\mathrm{PTS}} A: B$ then there exist some $s \in \mathcal{S}$ such that either $B=s$ or $\Gamma \vdash_{\mathrm{PTS}} B: s$.

Lemma 4 (Substitution [4, Lemma 5.2.11]). If $\Gamma[x: B] \vdash_{\text {PTS }} \alpha$ and $\Gamma \vdash_{\text {PTS }} A: B$ then $\Gamma \vdash_{\text {PTS }}\{A / x\} \alpha$.

Definition 2. A PTS is functional if $\left(\left\langle s_{1}, s\right\rangle \in \mathcal{A}\right.$ and $\left.\left\langle s_{1}, s^{\prime}\right\rangle \in \mathcal{A}\right)$ or $\left(\left\langle s_{1}, s_{2}, s\right\rangle \in \mathcal{R}\right.$ and $\left.\left\langle s_{1}, s_{2}, s^{\prime}\right\rangle \in \mathcal{R}\right)$ implies $s=s^{\prime}$.

Lemma 5 (Uniqueness [4, Lemma 5.2.21]). If a PTS is functional, $\Gamma \vdash_{\mathrm{PTS}} A: B$ and $\Gamma \vdash_{\mathrm{PTS}} A: B^{\prime}$ implies $B \stackrel{*}{\longleftrightarrow} B^{\prime}$.

In the following, we will only consider functional PTS.

\subsection{A scheme for $\lambda$-calculi with explicit substitu- tions}

Because we do not want that our work depends on a particular calculus with explicit substitution, we use a scheme, as is done in [18]. Our scheme is slightly different, because we use named variables and precooking [11], so that the $\lambda$-term $\lambda x(y x)$ is translated into $\lambda(y[$ shift $] \underline{1})$ instead of for instance $\lambda(\underline{2} \underline{1})$ using only De Bruijn indices. A pure term is therefore a term were free variables are correctly precooked. Notwithstanding, the results are the same in the two settings, provided we add conditions 10 and 11 below.

Definition 3 (Substitution calculus). A substitution calculus consists of a signature upon two sorts $\mathfrak{T}$ and $\mathfrak{S}$ which contains a constant shift : $\mathfrak{S}$, three function symbols $\lambda: \mathfrak{T} \hookrightarrow \mathfrak{T}, \cdots: \mathfrak{T} \times \mathfrak{T} \hookrightarrow \mathfrak{T}$ and $\cdot[\cdot]: \mathfrak{T} \times \mathfrak{S} \hookrightarrow \mathfrak{T}$, and in which can be defined two functions lift $: \mathfrak{S} \hookrightarrow \mathfrak{S}$ and cons : $\mathfrak{T} \hookrightarrow \mathfrak{S}$; and a sort-preserving rewrite system containing

$$
(a b)[s] \rightarrow a[s] b[s]
$$

(App)

and

$$
(\lambda a)[s] \rightarrow \lambda(a[\operatorname{lift}(s)])
$$

We also need the rule to trigger the $\beta$-reduction

$$
(\lambda a) b \rightarrow a[\operatorname{cons}(b)]
$$

which is not part of the rewrite system of the substitution calculus.

Definition 4 (Scheme). A substitution calculus $W$ obeys the scheme if 
Empty [] well-formed

Declaration $\frac{\Gamma \vdash_{\mathrm{PTS}} A: s}{\Gamma[x: A] \text { well-formed }} s \in \mathcal{S}$ and $x$ not in $\Gamma$

Sort $\frac{\Gamma \text { well-formed }}{\Gamma \vdash_{\mathrm{PTS}} s_{1}: s_{2}}\left\langle s_{1}, s_{2}\right\rangle \in \mathcal{A}$

Variable $\frac{\Gamma \text { well-formed } x: A \in \Gamma}{\Gamma \vdash_{\mathrm{PTS}} x: A}$

$$
\begin{array}{r}
\text { Product } \frac{\Gamma \vdash_{\mathrm{PTS}} A: s_{1} \quad \Gamma[x: A] \vdash_{\mathrm{PTS}} B: s_{2}}{\Gamma \vdash_{\mathrm{PTS}} \Pi x: A B: s_{3}}\left\langle s_{1}, s_{2}, s_{3}\right\rangle \in \mathcal{R} \\
\text { Application } \frac{\Gamma \vdash_{\mathrm{PTS}} T: \Pi x: A B \quad \Gamma \vdash_{\mathrm{PTS}} U: A}{\Gamma \vdash_{\mathrm{PTS}}(T U):\{U / x\} B} \\
\text { Abstraction } \frac{\Gamma \vdash_{\mathrm{PTS}} \Pi x: A B: s \quad \Gamma[x: A] \vdash_{\mathrm{PTS}} T: B}{\Gamma \vdash_{\mathrm{PTS}} \lambda x: A T: \Pi x: A B} \\
\text { Conversion } \frac{\Gamma \vdash_{\mathrm{PTS}} T: A \quad \Gamma \vdash_{\mathrm{PTS}} B: s}{\Gamma \vdash_{\mathrm{PTS}} T: B} s \in \mathcal{S} \text { and } A \stackrel{{ }^{*}}{\longleftrightarrow} B
\end{array}
$$

Figure 2. Typing rules of a PTS $\langle S, \mathcal{A}, \mathcal{R}\rangle$

1. $W$ is confluent

2. $W$ is strongly normalizing

3. $W$-normal forms of terms are pure terms

4. $W(a b)=W(a) W(b)$ and $W(\lambda a)=\lambda W(a)$, where $W(a)$ denotes the $W$-normal form of $a$

5. for every substitution $s: \mathfrak{S}$ in $W, \underline{1}[\operatorname{lift}(s)] \stackrel{*}{\longleftrightarrow} W \underline{1}$

6. for every substitution $s: \mathfrak{S}$ in $W$ and $m \geq 1$, $\underline{m+1}[\operatorname{lift}(s)] \stackrel{*}{\longleftrightarrow} W \underline{m}[s][$ shift $]$

7. for every term $k: \mathfrak{T}$ and $m \geq 1, \underline{m+1}[\operatorname{cons}(k)] \stackrel{*}{\longleftrightarrow} W \underline{m}$

8. for every term $k: \mathfrak{T}, \underline{1}[\operatorname{cons}(k)] \stackrel{*}{\longleftrightarrow} W k$

9. for every $m, \underline{m}[$ shift $] \stackrel{*}{\longleftrightarrow} W \underline{m+1}$

10. for every named variable $x$, substitution $s: \mathfrak{S}$, and $n \geq 0, x[\text { shift }]^{n+1}[\operatorname{lift}(s)] \stackrel{*}{\longleftrightarrow} x[\text { shift }]^{n}[s][$ shift $]$

11. for every named variable $x$, term $k: \mathfrak{T}$, and $n \geq 0$, $x[\text { shift }]^{n+1}[\operatorname{cons}(k)] \stackrel{*}{\longleftrightarrow} W[\text { shift }]^{n}$

12. for every function symbol $\xi: \mathfrak{K}_{1} \times \cdots \times \mathfrak{K}_{q} \rightarrow \mathfrak{S}$ and $\underline{m} \geq 1$, one of the two following conditions holds:

- there exists some $n$, distinct indices $i_{1}, \ldots, i_{p}$ in $\{1 ; \cdots ; q\}$ and substitutions $u_{1}, \ldots, u_{k}$ such that for all $s_{1}, \ldots, s_{q}$ we have $\underline{m}\left[\xi\left(q_{1}, \ldots, q_{n} s\right)\right] \stackrel{*}{\longleftrightarrow} W \underline{n}\left[s_{i_{1}}\right] \ldots\left[s_{i_{p}}\right]\left[u_{1}\right] \ldots\left[u_{k}\right] ;$

- there exists $i \in\{1 ; \cdots ; q\}$ such that for all $s_{1}, \ldots, s_{q}$ we have $\underline{m}\left[\xi\left(q_{1}, \ldots, q_{n} s\right)\right] \stackrel{*}{\longleftrightarrow} W s_{i}$.

In particular, these conditions implies that $\lambda_{W}$ $(\stackrel{!}{=} W+$ Beta $)$ is confluent [18, Theorem 5.18]. Many calculi with explicit substitution are instances of this scheme, in particular $\lambda_{\sigma}$ [1] (see [18] for others).

Note that we do not use the same parenthesis conventions for PTS terms and (first-order) terms of the scheme, in order to make a visual distinction between them.

\section{Superdeductive systems for intuitionistic logic}

\subsection{Supernatural deduction}

Supernatural deduction $\mathrm{LJ}^{+}$was introduced by Wack [23] as an alternative to deduction modulo [13]. It con-
Introduction rules: Elimination rules:

$$
\begin{array}{cc}
\frac{\Gamma \vdash A}{\Gamma \vdash A^{*}} & \frac{\Gamma \vdash A^{*}}{\Gamma \vdash A} \\
\frac{\Gamma \vdash \perp}{\Gamma \vdash \perp^{*}} & \frac{\Gamma \vdash \perp^{*}}{\Gamma \vdash C} \\
\frac{\Gamma, P \vdash Q^{*}}{\Gamma \vdash(P \Rightarrow Q)^{*}} & \frac{\Gamma \vdash(P \Rightarrow Q)^{*} \quad \Gamma \vdash P}{\Gamma \vdash Q^{*}} \\
\frac{\Gamma \vdash P^{*} \quad \Gamma \vdash Q^{*}}{\Gamma \vdash(P \wedge Q)^{*}} & \frac{\Gamma \vdash(P \wedge Q)^{*}}{\Gamma \vdash P^{*}} \frac{\Gamma \vdash(P \wedge Q)^{*}}{\Gamma \vdash Q^{*}} \\
\frac{\Gamma \vdash P^{*}}{\Gamma \vdash(\forall x . P)^{*}} x \text { not free in } \Gamma & \frac{\Gamma \vdash(\forall x . P)^{*}}{\Gamma \vdash(\{t / x\} P)^{*}}
\end{array}
$$

Figure 3. Rules for computing super introduction and elimination rules

sists in computing new inference rules that are adapted to the theory we are considering. For technical reasons, this theory has to be expressed as two rewrite systems. The former rewrites terms into terms, the latter rewrites atomic propositions to arbitrary formulæ. The idea is to shorten the proofs by precomputing the steps that naturally occur after the unfolding of a definition corresponding to the proposition rewrite system.

Supernatural deduction is based on natural deduction [14] and the new rules can be seen as introduction and elimination rules corresponding to the rewrite rule. Given a rewrite rule $A \rightarrow P$, its introduction rule is computed by applying from bottom to top all the introduction rules of figure 3 on $P^{*}$ while a $*$ remains. The premises of the new rule are then the remaining open leaves, whereas its conclusion is $A$, and we keep all the freshness conditions. Elimination rules are computed using the elimination rules of figure 3 from top left to bottom. This rules are then applied modulo the term rewrite system (see [13]). 
Example 1: Let $C r a b$ be the rule $A \rightarrow B \wedge(A \Rightarrow \perp)$.

The computation of the introduction rule is

$$
\frac{\Gamma \vdash B^{*} \quad \frac{\Gamma, A \vdash \perp^{*}}{\Gamma \vdash(A \Rightarrow \perp)^{*}}}{\Gamma \vdash(B \wedge(A \Rightarrow \perp))^{*}}
$$

and the new rule is therefore

$$
\operatorname{Crab}_{I} \frac{\Gamma \vdash^{+} B \quad \Gamma, A \digamma^{+} \perp}{\Gamma \vdash^{+} A}
$$

The computation of the elimination rules are

$$
\frac{\Gamma \vdash(B \wedge(A \Rightarrow \perp))^{*}}{\Gamma \vdash B^{*}} \text { and } \quad \frac{\frac{\Gamma \vdash(B \wedge(A \Rightarrow \perp))^{*}}{\Gamma \vdash(A \Rightarrow \perp)^{*}} \quad \Gamma \vdash A}{\frac{\Gamma \vdash \perp^{*}}{\Gamma \vdash C}}
$$

and the new rules are therefore

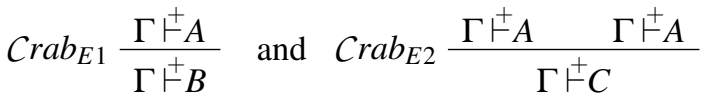

\subsection{Super sequent calculus}

The super sequent calculus was introduced by Brauner, Houtmann and Kirchner [8] as the analog of supernatural deduction for the sequent calculus for classical logic. We propose here its counterpart for intuitionistic logic. It is based on LB (see section 2.1). The idea to compute the left (resp. right) rule for a rewrite rule $A \rightarrow P$ is to apply LB rules on $\Gamma, P^{*} \vdash \Delta$ (resp. $\Gamma \vdash P^{*}, \Delta$ ) unless there is a permutation problem. Rules for the computation of super sequent rules $\mathrm{LB}^{+}$are summed up in figure 4 . Once again, the new inference rules are applied modulo the term rewrite system. Example 2: We consider the rewrite rule Crab of example 1. The computation of the left rule is

$$
\frac{\overline{\Gamma, B, \perp^{*} \vdash \Delta} \quad \Gamma, B \vdash A^{*}, \Delta}{\frac{\Gamma, B^{*},(A \Rightarrow \perp)^{*} \vdash \Delta}{\Gamma,(B \wedge(A \Rightarrow \perp))^{*} \vdash \Delta}}
$$

and the new rule is therefore

$$
\operatorname{Crab}_{L} \frac{\Gamma, B \stackrel{\vdash}{+}^{+}, \Delta}{\Gamma, A \vdash^{+} \Delta}
$$

The computation of the right rule is

$$
\frac{\Gamma \vdash B^{*}, \Delta \quad \frac{\Gamma, A \vdash \perp^{*}}{\Gamma \vdash(A \Rightarrow \perp)^{*}, \Delta}}{\Gamma \vdash(B \wedge(A \Rightarrow \perp))^{*}, \Delta}
$$

and the new rule is therefore
Left rules: $\quad$ Right rules

$$
\begin{array}{ll}
\frac{\Gamma, A \vdash \Delta}{\Gamma, A^{*} \vdash \Delta} & \frac{\Gamma \vdash A, \Delta}{\Gamma \vdash A^{*}, \Delta} \\
\hline \Gamma, \perp^{*} \vdash \Delta & \frac{\Gamma \vdash \perp, \Delta}{\Gamma \vdash \perp^{*}, \Delta}
\end{array}
$$

$$
\begin{array}{cc}
\frac{\Gamma, Q^{*} \vdash \Delta}{\Gamma,(P \Rightarrow Q)^{*} \vdash \Delta} & \frac{\Gamma, P \vdash Q^{*}}{\Gamma \vdash(P \Rightarrow Q)^{*}, \Delta} \\
\frac{\Gamma, P^{*}, Q^{*} \vdash \Delta}{\Gamma,(P \wedge Q)^{*} \vdash \Delta} & \frac{\Gamma \vdash P^{*}, \Delta \quad \Gamma \vdash Q^{*}, \Delta}{\Gamma \vdash(P \wedge Q)^{*}, \Delta} \\
\frac{\Gamma,(\{t / x\} P)^{*} \vdash \Delta}{\Gamma,(\forall x . P)^{*} \vdash \Delta} & \frac{\Gamma \vdash P^{*}}{\Gamma \vdash(\forall x . P)^{*}, \Delta} x \text { not free in } \Gamma
\end{array}
$$

\section{Figure 4. Rules for computing super left and right rules}

$$
\operatorname{Crab}_{R} \frac{\Gamma \vdash^{+} B, \Delta \quad \Gamma, A \digamma^{+} \perp}{\Gamma \vdash^{+} A, \Delta}
$$

We also introduce the notion of permutation-problem free formulæ, for which we will prove that the atomic super sequent calculus is complete:

Definition 5 (Permutation-problem freeness). The set of permutation-problem free formulæ (ppf formula) is build on the the following grammar:

$$
P \stackrel{!}{=} A|\perp| A \Rightarrow P|P \wedge P| \forall x . P
$$

where A denotes an atomic proposition.

A rewrite rule is said to be ppf if its right-hand side is ppf, and so does a rewrite system if all its rules are.

Proposition 6 (Atomic $\mathrm{LB}^{+}$). If the rewrite system is ppf, and $\Gamma$ and $\Delta$ contains only atomic formula, then $\Gamma \vdash \Delta$ is provable iff it is provable with only identity and super rules.

Proof. The idea is to use a procedure similar to the cut elimination, which transforms cuts around a formula with a connector into cuts around subformulæ. This shows that we only need atomic cuts. Then, remark that the rewrite system is ppf iff the new left and right rules contains only atomic propositions. Thus, starting with atomic propositions, we can only apply atomic cuts or super rules, and these do not introduce non-atomic propositions. More details can be found in the section A.1.

\subsection{Translations between these systems}

The proofs of this section can be found in appendix A. 


\section{THEOREM 7.}

For a given rewrite system, any sequent provable in $\mathrm{NJ}^{+}$ can be proved in $\mathrm{LB}^{+}$.

Corollary 8. A sequent is provable in $\mathrm{LB}^{+}$if it is provable in LB adding a compatible theory in the sense of [13, Definition 1.4] in the hypotheses.

Proof. Wack [23] proved that $\mathrm{NJ}^{+}$is equivalent to $\mathrm{NJ}$ with assumptions in the compatible theory, and we can translate every proof of LB into $\mathrm{NJ}$, as well as proofs in $\mathrm{NJ}^{+}$into proofs of $\mathrm{LB}^{+}$.

Note: $\mathrm{LB}^{+}$without the cut rule may be incomplete in that sense, as shown by the example of the $C r a b$ rule. In $\mathrm{LB}^{+}$ there is no cut-free proof of $B \vdash \perp$ but we can build the first proof of figure 5 with cuts.

\section{THEOREM 9.}

A proof of a sequent in $\mathrm{LB}^{+}$using only identity and super rules can be transformed into a proof of the same sequent in $\mathrm{NJ}^{+}$using only super rules and Axiom.

Corollary 10. For ppf rewrite systems, sequents containing only atomic propositions can be proved in $\mathrm{NJ}^{+}$iff they do using only super rules and Axiom.

Note: The admissibility of the cut rule in the super sequent calculus is not needed for the completeness of the atomic supernatural deduction. For instance, for the rewrite rule Crab, the first proof of $B \vdash \perp$ in $\mathrm{LB}^{+}$in figure 5 is translated into the second proof in $\mathrm{NJ}^{+}$which indeed contains only atomic formulæ.

\section{PTS as superdeductive theories}

In this section, we consider some functional PTS $\langle S, \mathcal{A}, \mathcal{R}\rangle$.

\subsection{The system}

The term rewrite rules correspond to some $\lambda$-calculus with explicit substitutions $\lambda_{W}$. As we do not want to rely on a particular calculus, we use the scheme proposed by [18]. See section 2.3.

We also use some function symbols to represent the $\Pi$ binder of the PTS. As done in [9], we need a new function symbol $\dot{\pi}_{\left\langle s_{1}, s_{2}, s_{3}\right\rangle}$ for each rule $\left\langle s_{1}, s_{2}, s_{3}\right\rangle \in \mathcal{R}$. We need to tell how they interact with explicit substitutions by adding term rewrite rules:

$$
\dot{\pi}_{\left\langle s_{1}, s_{2}, s_{3}\right\rangle}(a, b)[s] \rightarrow \dot{\pi}_{\left\langle s_{1}, s_{2}, s_{3}\right\rangle}(a[s], b[\operatorname{lift}(s)]) .
$$

Note that we use lift on the second argument as is done for $\lambda$ in (Lambda), because some variable is bound by $\dot{\pi}_{\left\langle s_{1}, s_{2}, s_{3}\right\rangle}$.

$$
\begin{aligned}
& \text { (2) } E 1 \frac{\Gamma^{+} \varepsilon\left(\dot{\pi}_{\left\langle s_{1}, s_{2}, s_{3}\right\rangle}(a, b), s_{3}\right)}{\Gamma^{+} \varepsilon\left(a, s_{1}\right)} \\
& (2)_{E 2} \frac{\Gamma \vdash^{+} \varepsilon\left(\dot{\pi}_{\left\langle s_{1}, s_{2}, s_{3}\right\rangle}(a, b), s_{3}\right) \quad \Gamma \vdash^{+} \varepsilon(u, a)}{\Gamma \vdash^{+} \varepsilon\left(b[\operatorname{cons}(u)], s_{2}\right)} \\
& \text { (3) } E 1 \frac{\Gamma^{+} \varepsilon\left(t, \dot{\pi}_{\left\langle s_{1}, s_{2}, s_{3}\right\rangle}(a, b)\right)}{\Gamma{ }^{+} \varepsilon\left(\dot{\pi}_{\left\langle s_{1}, s_{2}, s_{3}\right\rangle}(a, b), s_{3}\right)}
\end{aligned}
$$

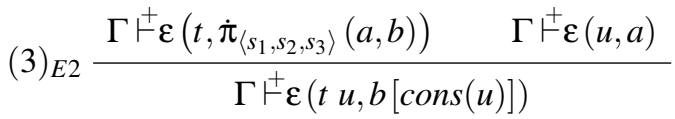

\section{Figure 7. Elimination rules of the $\mathrm{NJ}^{+}$systems for the PTS}

We also need a constant $\mathrm{s}$ for each sort $s \in \mathcal{S}$. We add the following term rewrite rules, which are not necessary but permit to avoid precooking the $s$ constants in the translation from PTS terms to first-order terms (see below):

$$
\mathrm{s}[t] \rightarrow \mathrm{s} .
$$

In the following, $\longrightarrow$ designates the rewrite relation associated with the term rewrite system we have just defined.

Let $\varepsilon: \mathfrak{T} \times \mathfrak{T}$ be a predicate. $\varepsilon(a, b)$ should be seen as $a$ has type $b$. The proposition rewrite rules permits to simulate the inference rules of the PTS:

$$
\begin{aligned}
\varepsilon\left(\mathrm{s}_{1}, \mathrm{~s}_{2}\right) \rightarrow & \top \quad\left(\left\langle s_{1}, s_{2}\right\rangle \in \mathcal{A}\right)(1) \\
\varepsilon\left(\dot{\pi}_{\left\langle s_{1}, s_{2}, s_{3}\right\rangle}(a, b), \mathrm{s}_{3}\right) \rightarrow & \varepsilon\left(a, \mathrm{~s}_{1}\right) \wedge \quad(2) \\
& \forall z \cdot \varepsilon(z, a) \Rightarrow \varepsilon\left(b[\operatorname{cons}(z)], \mathrm{s}_{2}\right) \\
\varepsilon\left(t, \dot{\pi}_{\left\langle s_{1}, s_{2}, s_{3}\right\rangle}(a, b)\right) \rightarrow & \varepsilon\left(\dot{\pi}_{\left\langle s_{1}, s_{2}, s_{3}\right\rangle}(a, b), s_{3}\right) \wedge \quad(3) \\
& \forall z \cdot \varepsilon(z, a) \Rightarrow \varepsilon(t z, b[\operatorname{cons}(z)])
\end{aligned}
$$

These rewrite rules leads to the inference rules in supernatural deduction represented in figures 6 and 7 .

We can now define a translation from PTS terms (i.e. well-typed pseudo terms) into our first-order terms. This translation is inspired from the one between $\lambda$-calculus with de Bruijn indices and $\lambda_{\sigma}$ with precooking [11, Definition 4.1], except that we translate directly from a $\lambda$-calculus with names, and we deal with the binder $\Pi$. $\bar{\ell}$ means the length of the list $\ell$ in the usual sense.

Definition 6 (Translation). The translation takes as parameters a context $\Gamma$ and a list $\ell$ (which are omitted if unchanged):

$$
\begin{array}{rlr}
|x|_{\ell_{1}:: x: \ell_{2}} & \stackrel{!}{=} \overline{\ell_{1}}+1 & \text { if } x \notin \ell_{1} \\
|x|_{\ell} & \stackrel{!}{=} x[\text { shift }]^{\bar{\ell}} & \text { if } x \notin \ell
\end{array}
$$




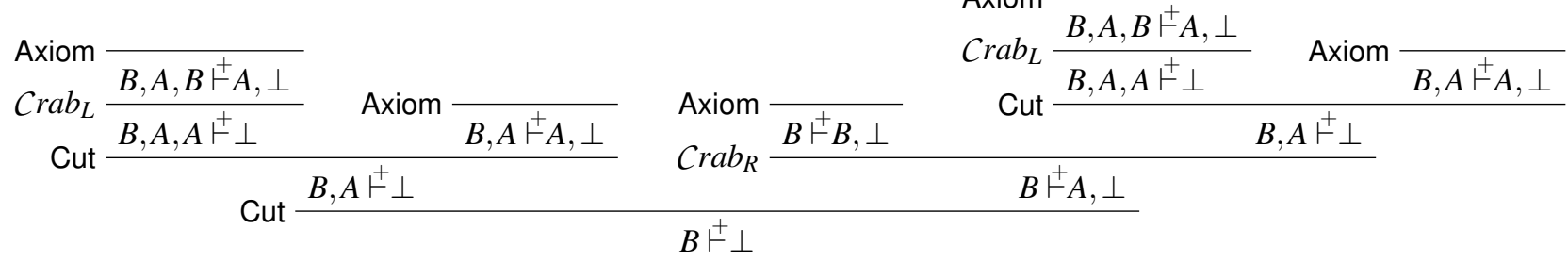

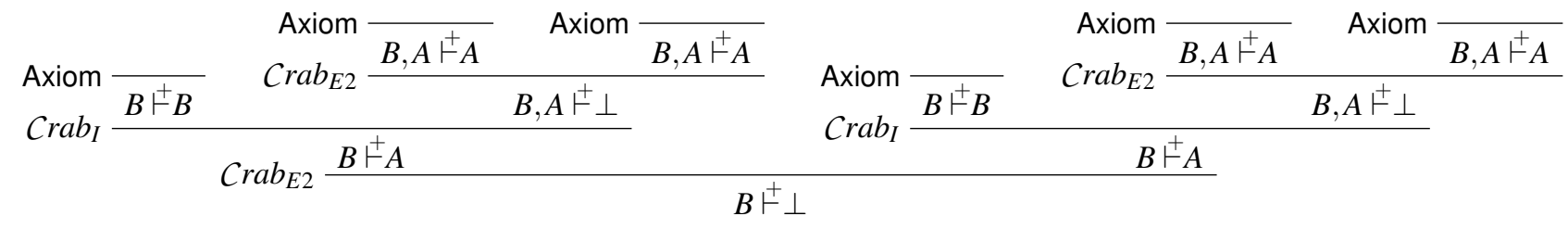

Figure 5. Example of the non-admissibility of cut in $\mathrm{LB}^{+}$and its translation in $\mathrm{NJ}^{+}$

$$
\begin{gathered}
(1)_{I} \frac{(2)_{I} \frac{\Gamma \vdash^{+} \varepsilon\left(a, s_{1}\right)}{\Gamma \digamma^{+} \varepsilon\left(s_{1}, s_{2}\right)}\left\langle s_{1}, s_{2}\right\rangle \in \mathcal{A} \quad \Gamma(z, a) \vdash^{+} \varepsilon\left(b[\operatorname{cons}(z)], s_{2}\right)}{\Gamma \vdash^{+} \varepsilon\left(\dot{\pi}_{\left\langle s_{1}, s_{2}, s_{3}\right\rangle}(a, b), s_{3}\right)} z \text { not free in } \Gamma,\left\langle s_{1}, s_{2}, s_{3}\right\rangle \in \mathcal{R} \\
(3)_{I} \frac{\Gamma{ }^{+} \varepsilon\left(\dot{\pi}_{\left\langle s_{1}, s_{2}, s_{3}\right\rangle}(a, b), s_{3}\right)}{\Gamma{ }^{+} \varepsilon\left(t, \dot{\pi}_{\left\langle s_{1}, s_{2}, s_{3}\right\rangle}(a, b)\right)} z, \varepsilon(z, a) \vdash^{+} \varepsilon(t z, b[\operatorname{cons}(z)]) \\
\text { not free in } \Gamma,\left\langle s_{1}, s_{2}, s_{3}\right\rangle \in \mathcal{R}
\end{gathered}
$$

Figure 6. Introduction rules of the $\mathrm{NJ}^{+}$systems for the PTS

$$
\begin{aligned}
|s|_{\ell} & \stackrel{!}{=} \mathrm{s} \\
|\lambda x: A T|_{\ell}^{\Gamma} & \stackrel{!}{=} \lambda|T|_{x:: \ell}^{\Gamma[x: A]} \\
|A B| & \stackrel{!}{=}|A||B| \\
|\Pi x: A B|_{\ell}^{\Gamma} & \stackrel{!}{=} \dot{\pi}_{\left\langle s_{1}, s_{2}, s_{3}\right\rangle}\left(|A|_{\ell}^{\Gamma},|B|_{x:: \ell}^{\Gamma[x: A]}\right)
\end{aligned}
$$

where $s_{1}$ corresponds the type of $A$ in $\Gamma, s_{2}$ the type of $B$ in $\Gamma[x: A]$, and $\left\langle s_{1}, s_{2}, s_{3}\right\rangle \in \mathcal{R}$.

Then, a context is translated inductively by $|[]| \stackrel{!}{=} \emptyset$ and $|\Gamma[x: A]| \stackrel{!}{=}|\Gamma|, \varepsilon\left(x,|A|_{[]}^{\Gamma}\right)$, and a typing judgment $\Gamma \vdash_{\text {PTS }} A: B$ is translated into $|\Gamma| \vdash^{+} \varepsilon\left(|A|_{[]}^{\Gamma},|B|_{[]}^{\Gamma}\right)$. In the following, contexts and lists will be omitted if they can be deduce from the environment.

Note that this translation is deterministic, because the PTS is assumed to be functional. Note also that if $\Gamma \subseteq \Gamma^{\prime}$ then $|T|^{\Gamma}=|T|^{\Gamma^{\prime}}$ if they are defined, by [4, Lemma 5.2.12].

\subsection{Soundness and conservativeness}

We need the four following lemmata to prove the soundness and the conservativeness of the $\mathrm{NJ}^{+}$system w.r.t. the PTS. Their proofs can be found in appendix B.

Lemma 11. $|B|_{x}\left[\operatorname{cons}\left(|U|_{[]}\right)\right] \stackrel{*}{\longleftrightarrow}|\{U / x\} B|_{[]}$.
Lemma 12. If $|A| \stackrel{*}{\longrightarrow}$ a then there exists $A^{\prime}$ such that $a \stackrel{*}{\longrightarrow}\left|A^{\prime}\right|$.

Lemma 13. $|B|_{z}=\{\underline{1} / z[$ shift $]\}\left(|B|_{[]}[\right.$shift $\left.]\right)$

Lemma 14 (Reduction under $\Pi$ ). - If $\left\langle s_{1}, s_{2}, s_{3}\right\rangle \in \mathcal{R}$, $\Gamma \vdash_{\mathrm{PTS}} A: s_{1}$ and $\Gamma[x: A] \vdash_{\mathrm{PTS}} B: s_{2}, \quad x$ is not free in $b, a \stackrel{*}{\longrightarrow}|A|^{\Gamma}$ and $b[\operatorname{cons}(x)] \longrightarrow|B|^{\Gamma[x: A]}$ then $\dot{\pi}_{\left\langle s_{1}, s_{2}, s_{3}\right\rangle}(a, b) \stackrel{*}{\longleftrightarrow}|\Pi x: A B|^{\Gamma}$.

- If $\dot{\pi}_{\left\langle s_{1}, s_{2}, s_{3}\right\rangle}(a, b) \stackrel{*}{\longrightarrow}|C|$ then there exists $A, B$ such that $C=\Pi x: A B$.

THEOREM 15 (Soundness).

For all PTS terms $T$ and $A$, if $\Gamma \vdash_{\mathrm{PTS}} T: A$ then $|\Gamma| \vdash^{+} \varepsilon(|T|,|A|)$.

Proof. The typing rules of the PTS, represented on figure 2, are translated into the corresponding derivations in $\mathrm{NJ}^{+}$represented on figure 8.

Some comments: The $\equiv$ derivations are not really inference rules, but corresponds to the fact that we are working modulo the term rewrite system $\lambda_{W}$; they permit to let the computation appears. $\Gamma^{\prime}$ in the translation of Abstraction is $|\Gamma|, \varepsilon(z,|A|)$. In the translation of Conversion, we do not check the type of the converts; this is not problematic as shown by the conservativeness result below. In the 
Empty:

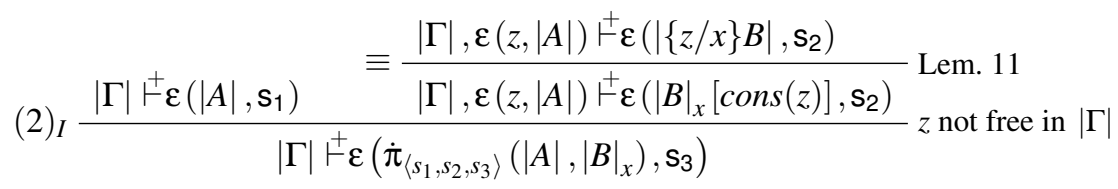

Declaration: we do not need to look at the wellformedness of the context

$$
(3)_{E 2} \frac{|\Gamma| \vdash^{+} \varepsilon\left(|T|, \dot{\pi}_{\left\langle s_{1}, s_{2}, s_{3}\right\rangle}\left(|A|,|B|_{x}\right)\right) \quad|\Gamma| \vdash^{+} \varepsilon(|U|,|A|)}{\equiv \frac{|\Gamma| \vdash^{+} \varepsilon\left(|T||U|,|B|_{x}[\operatorname{cons}(|U|)]\right)}{|\Gamma| \vdash^{+} \varepsilon(|T||U|,|\{U / x\} B|)} \text { Lem. } 11}
$$

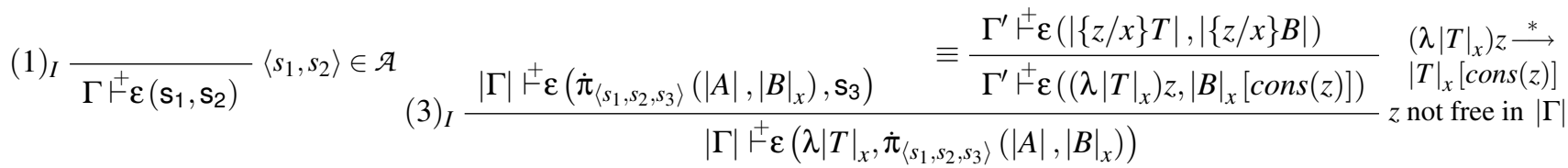

Axiom $\overline{|\Gamma| \digamma^{+} \varepsilon(x,|A|)}$

Conversion: using $\lambda_{W}$ in the modulo

\section{Figure 8. Derivations in $\mathrm{NJ}^{+}$corresponding to the typing rules of a PTS (see figure 2)}

translation of Variable, $\varepsilon(x,|A|)$ is in $|\Gamma|$ by definition of the translation of a context. In the translations of Abstraction and Product, we use the fact that $z$ is free in $|\Gamma|$ to translate proofs of $\Gamma[x: A] \vdash_{\text {PTS }} \alpha$ into proofs of $\Gamma[z: A] \vdash_{\text {PTS }}\{z / x\} \alpha$. In the translation of Abstraction, we know that $|s|=s_{3}$ by Lemma 5.

THEOREM 16 (Conservativeness).

For all well-formed context $\Gamma$, for all terms $a, b$ if $|\Gamma| \vdash^{+} \varepsilon(a, b)$ then there exists $A$ and $B$ such that $a \stackrel{*}{\longrightarrow}|A|^{\Gamma}, b \stackrel{*}{\longrightarrow}|B|^{\Gamma}$ and $\Gamma \vdash_{\mathrm{PTS}} A: B$.

Proof. By induction on the proof of $|\Gamma| \vdash^{+} \varepsilon(a, b)$ and the number of uses of the modulo on terms.

If the last rule is applied modulo the term rewrite system, we apply it without modulo and then consider the modulo:

$$
\equiv \frac{|\Gamma|{ }^{+} \varepsilon\left(a^{\prime}, b^{\prime}\right)}{\left.|\Gamma|\right|^{+} \varepsilon(a, b)} \quad \begin{aligned}
& a \stackrel{*}{\longleftrightarrow} a^{\prime} \\
& b \stackrel{*}{\longleftrightarrow} b^{\prime}
\end{aligned}
$$

By induction hypothesis, there exists some $A^{\prime}$ and $B^{\prime}$ such that $a^{\prime} \stackrel{*}{\longrightarrow}\left|A^{\prime}\right|, b^{\prime} \stackrel{*}{\longrightarrow}\left|B^{\prime}\right|$ and $\Gamma \vdash_{\mathrm{PTS}} A^{\prime}: B^{\prime}$. By confluence of $\lambda_{W}$, there exists $a^{\prime \prime}$ such that $a \stackrel{*}{\longrightarrow} a^{\prime \prime} \stackrel{*}{\longleftarrow}\left|A^{\prime}\right|$. By Lemma 12 there exists some $A$ such that $a^{\prime \prime} \stackrel{*}{\longrightarrow}|A|$. By subject reduction, $\Gamma \vdash_{\mathrm{PTS}} A: B^{\prime}$. By confluence of $\lambda \sigma$, there exists $b^{\prime \prime}$ such that $b \stackrel{*}{\longrightarrow} b^{\prime \prime} \stackrel{*}{\longleftarrow}\left|B^{\prime}\right|$. By Lemma 12 there exists $B$ such that $b^{\prime \prime} \stackrel{*}{\longrightarrow}|B|$. By Lemma 3, either $B^{\prime}$ is a sort, in which case it is in normal form and $B=B^{\prime}$, or there exists some sort $s \in \mathcal{S}$ such that $\Gamma \vdash_{\mathrm{PTS}} B^{\prime}: s$, and by subject reduction $\Gamma \vdash_{\text {PTS }} B: s$. Therefore, using Conversion we deduce that $\Gamma \vdash_{\mathrm{PTS}} A: B$. In the following we can therefore assume that the inference rules are applied without modulo. We use the names of the variables used on the fig, 6 and 7 .
- If the last rule is an axiom, hence of the form:

$$
\text { Axiom } \overline{\left|\Gamma_{1}\right|, \varepsilon(x, b),\left.\left|\Gamma_{2}\right|\right|^{+} \varepsilon(x, b)}
$$

By definition of the translation of contexts, this means that $b=|B|$ with $x: B \in \Gamma$. Because by assumption $\Gamma$ is wellformed, we can therefore use Variable to get a proof of $\Gamma \vdash_{\text {PTS }} x: B$.

- If the last rule is $(1)_{I}:$ as $\Gamma$ is assumed to be wellformed, we can apply Sort.

- If the last rule is $(2)_{I}$ : By induction hypothesis on the left subproof, there exists $A$ such that $a \stackrel{*}{\longrightarrow}|A|$ and $\Gamma \vdash_{\mathrm{PTS}} A: s$. As $z$ is free in $\Gamma, \Gamma[z: A]$ is well-formed. Therefore we can use the induction hypothesis on the right subproof: there exists $B$ such that $b[\operatorname{cons}(z)] \stackrel{*}{\longrightarrow}|B|$ and $\Gamma[z: A] \vdash_{\mathrm{PTS}} B: s_{2}$. We can consider that $z$ is free in $b$, hence by Lemma $14 \dot{\pi}_{s}(a, b) \stackrel{*}{\longleftrightarrow}|\Pi z: A B|$, so we can conclude using the confluence of $\lambda_{W}$, subject reduction and Product.

- If the last rule is $(3)_{I}$ : By induction hypothesis on the left subproof, there exists $C$ such that $\Gamma{ }_{\mathrm{PTS}} C: s_{3}$ and $\dot{\pi}_{\left\langle s_{1}, s_{2}, s_{3}\right\rangle}(a, b) \stackrel{*}{\longrightarrow}|C|$. By Lemma 14 there exists $A, B_{1}$ such that $C=\Pi x: A B_{1}$. Because $\lambda_{W}$ cannot change the function symbols $\dot{\pi}_{\left\langle s_{1}, s_{2}, s_{3}\right\rangle}$, that means that $|C|=\dot{\pi}_{\left\langle s_{1}, s_{2}, s_{3}\right\rangle}\left(|A|,\left|B_{1}\right|_{x}\right), a \stackrel{*}{\longrightarrow}|A|$ and $b \stackrel{*}{\longrightarrow}\left|B_{1}\right|_{x}$. Hence $\Gamma \vdash_{\text {PTS }} A: s_{1}$, and the context $\Gamma[z: A]$ is wellformed because $z$ is free in $|\Gamma|$. By induction hypothesis on the right subproof, there exists $T^{\prime}, B_{2}$ such that $\Gamma[z: A] \vdash_{\mathrm{PTS}} T^{\prime}: B_{2}$ with $t z \stackrel{*}{\longrightarrow}\left|T^{\prime}\right|$ and $b[\operatorname{cons}(z)] \stackrel{*}{\longrightarrow}\left|B_{2}\right|$. By Lemma $11\left|\{z / x\} B_{1}\right| \stackrel{*}{\longleftrightarrow}\left|B_{2}\right|$, hence by confluence of $\lambda_{W}$ and Lemma 12 there is a $B$ such that $|B|$ is a common reduct. By $\alpha$-conversion and subject reduction $\Gamma \vdash_{\mathrm{PTS}} \Pi z: A B: s_{3}$ and using Conversion $\Gamma[z: A] \vdash_{\mathrm{PTS}} T^{\prime}: B$. 
Using Abstraction we obtain $\Gamma \vdash_{\mathrm{PTS}} \lambda z: A T^{\prime}: \Pi z: A B$. By Lemma $14, \dot{\pi}_{\left\langle s_{1}, s_{2}, s_{3}\right\rangle}(a, b) \stackrel{*}{\longrightarrow} \Pi z: A B$.

Now consider the reduction $t z \stackrel{*}{\longrightarrow}\left|T^{\prime}\right|$. There are two cases: Either the outermost application never reduces, and therefore $\left|T^{\prime}\right|=t_{1} z$. In that case, by definition of the translation, we must have $T^{\prime}=(T z)$ for some $T$ with $t \stackrel{*}{\longrightarrow}|T|$. By subject reduction for $\eta$, from $\Gamma \vdash_{\text {PTS }} \lambda z: A(T z): \Pi z: A B$ we obtain $\Gamma \vdash_{\mathrm{PTS}} T: \Pi z: A B$.

In the other case, we have $t z \stackrel{*}{\longrightarrow}\left(\lambda t_{1}\right) z \stackrel{*}{\longrightarrow}\left|T^{\prime}\right|$. Because $z$ is not free in $\Gamma$, we can consider it is not free in $t$. Therefore $\lambda(t[$ shift $] \underline{1})=\{\underline{1} / z[$ shift $]\} \lambda(t[$ shift $] z[$ shift $])$ $=\lambda(\{\underline{1} / z[$ shift $]\}((t z)[$ shift $]))$$$
\stackrel{*}{\longrightarrow} \lambda\left(\{\underline{1} / z[\text { shift }]\}\left(\left|T^{\prime}\right|_{[]}[\text {shift }]\right)\right)
$$$$
\underset{\text { Lem. 13 }}{\stackrel{*}{\longrightarrow}} \lambda\left|T^{\prime}\right|_{z}=\left|\lambda z: A T^{\prime}\right|
$$

$$
\begin{array}{rll}
\operatorname{But} \lambda(t[\text { shift }] \underline{1}) & \stackrel{*}{\longrightarrow} & \lambda\left(\left(\lambda t_{1}\right)[\text { shift }] \underline{1}\right) \\
& \stackrel{*}{\longrightarrow} & \lambda\left(\left(\lambda t_{1}[\text { lift }(\text { shift })]\right) \underline{1}\right) \\
& \underset{(\text { Beta })}{\longrightarrow} & \lambda\left(t_{1}[\text { lift }(\text { shift })][\text { cons }(\underline{1})]\right) \\
& \lambda t_{1} \stackrel{*}{\longleftarrow} t
\end{array}
$$

Therefore $t \stackrel{*}{\longleftrightarrow}\left|\lambda z: A T^{\prime}\right|$ and by confluence of $\lambda_{W}$ and Lemma 12, there exists $T$ such that $t \stackrel{*}{\longrightarrow}|T|$ and $\lambda z: A T^{\prime} \stackrel{*}{\longrightarrow} \beta T$. We conclude by subject reduction.

- If the last rule is $(2)_{E 1}$ : By induction hypothesis, there exists $T$ such that $\dot{\pi}_{\left\langle s_{1}, s_{2}, s_{3}\right\rangle}(a, b) \stackrel{*}{\longrightarrow}|T|$ and $\Gamma \vdash_{\mathrm{PTS}} T: s_{3}$. By Lemma 14, there exists $A, B$ such that $T=\Pi x: A B$ and $a \stackrel{*}{\longrightarrow}|A|$. Then by definition of the translation, $|T|=$ $\dot{\pi}_{\left\langle s_{1}^{\prime}, s_{2}^{\prime}, s_{3}^{\prime}\right\rangle}\left(a^{\prime}, b^{\prime}\right)$ where $s_{1}^{\prime}$ is the type of $A$ in $\Gamma$, which exists because $\Gamma \vdash_{\text {PTS }} \Pi x: A B: s_{3}$. But the $\lambda_{W}$ reductions cannot change the function symbol $\dot{\pi}_{\left\langle s_{1}, s_{2}, s_{3}\right\rangle}$, hence $s_{1}=s_{1}^{\prime}$ and $\Gamma \vdash_{\mathrm{PTS}} A: s_{1}$.

- If the last rule is $(2)_{E 2}$ or $(3)_{E 1}$ : this comes from the definition of the translation, as in the previous case. We also need Lemma 4 for $(2)_{E 2}$.

- If the last rule is $(3)_{E 3}$ : Using the same techniques as in the previous cases, by induction hypothesis, we get proofs of $\Gamma \vdash_{\mathrm{PTS}} T: \Pi x: A B$ and $\Gamma \vdash_{\mathrm{PTS}} U: A$ for some $A, B, T, U$ such that $a \stackrel{*}{\longrightarrow}|A|, b \stackrel{*}{\longrightarrow}|B|, t \stackrel{*}{\longrightarrow}|T|$ and $u \stackrel{*}{\longrightarrow}|U|$ Using Application we get $\Gamma \vdash_{\text {PTS }}(T U):\{U / x\} B$. We can conclude because $t u \stackrel{*}{\longrightarrow}|(T U)|$ and by Corollary $11 b[\operatorname{cons}(u)] \stackrel{*}{\longrightarrow}|\{U / x\} B|$.

- If the last rule is another (i.e. logical) rule: we do not need this case because of Corollary 10.

Note: Because terms are $\beta$-convertible on the left of $\varepsilon(\cdot, \cdot)$ too, there is no correspondence between the term on the left of $\varepsilon(\cdot, \cdot)$ and the proof in $\mathrm{NJ}^{+}$. Nevertheless, the former is $\beta$-convertible to a term corresponding to the latter. This also implies that we cannot hope for a stronger result, since for instance we can prove $F^{+} \varepsilon((\lambda \lambda \underline{1})(\lambda \underline{1}) \star, \square)$ in supernatural deduction whereas it is not possible to prove ${ }_{\lambda \Pi}((\lambda x: ? \lambda y$ : ? $x \lambda x: ?(x x)) *): \square$ in the PTS $\lambda \Pi$.

But, for Theorem 16, if the PTS is strongly normalizing, then $A$ and $B$ are too, so $a$ and $b$ are weakly normalizing and we can reach some $\left|A^{\prime}\right|$ and $\left|B^{\prime}\right|$ from $a$ and $b$ such that $\Gamma \vdash_{\text {PTS }} A^{\prime}: B^{\prime}$ by simulating leftmost $\beta$-reduction.

\section{Applications}

\subsection{Using $\lambda_{\sigma}$ for the explicit-substitution calculus}

Because $\lambda_{\sigma}[1]$ is an instance of the scheme (see [18]), we can use it as $\lambda$-calculus with explicit substitutions. If we do so, Lemma 11 becomes

Lemma 17. $|B|_{x}\left[|U|_{[]} \cdot i d\right] \stackrel{*}{\longrightarrow}|\{U / x\} B|_{[]}$

It means that in the proof of soundness, rewriting is performed from bottom to top after an introduction rule, and from top to bottom after an elimination rules. If we translate such a proof into $\mathrm{LB}^{+}$using Theorem 7 , terms are rewritten only from the bottom to the top of the proof. This corresponds to the asymmetric sequent calculus modulo of Dowek [10]. This is also what is actually implemented in the proof assistant based on superdeduction Lemuridæ [8], where terms can only be reduced when building the proof tree from bottom to top.

\subsection{A sequent calculus for Pure Types Systems}

By applying Theorem 7 we obtain a sequent calculus presentation of Pure Type Systems. We do not detail all super left and right rules - that can be computed by the reader using figure 4-but the rules for (3):

$$
\begin{gathered}
\Gamma, \varepsilon\left(\dot{\pi}_{\left\langle s_{1}, s_{2}, s_{3}\right\rangle}(a, b), s_{3}\right), \varepsilon(t u, b[\operatorname{cons}(u)]) \vdash^{+} \Delta \\
(3)_{L} \frac{\Gamma, \varepsilon\left(\dot{\pi}_{\left\langle s_{1}, s_{2}, s_{3}\right\rangle}(a, b), s_{3}\right) \vdash^{+} \varepsilon(u, a), \Delta}{\Gamma, \varepsilon\left(t, \dot{\pi}_{\left\langle s_{1}, s_{2}, s_{3}\right\rangle}(a, b)\right) \vdash^{+} \Delta} \\
\Gamma{ }^{+} \varepsilon\left(\dot{\pi}_{\left\langle s_{1}, s_{2}, s_{3}\right\rangle}(a, b), s_{3}\right), \Delta \\
(3)_{R} \frac{\Gamma, \varepsilon(z, a) \vdash^{+} \varepsilon(t z, b[\operatorname{cons}(z)])}{\Gamma{ }^{+} \varepsilon\left(t, \dot{\pi}_{\left\langle s_{1}, s_{2}, s_{3}\right\rangle}(a, b)\right), \Delta}
\end{gathered}
$$

We can see how $(3)_{L}$ can be used to apply a function $t$ to an argument $u$.

Sequent calculi for PTS were already introduced by Gutiérrez and Ruiz [16] and by Lengrand et al. [19]. It remains to be investigated how the superdeductive system relates to these, what does not seem trivial. 


\section{Conclusion}

We have defined an natural encoding from functional PTS into supernatural deduction. We can therefore characterize PTS as first-order theories. We have proved the correctness and conservativeness of the translation, leading to a correspondence between valid typing judgments in the PTS and $\beta$-reducts of provable sequents in supernatural deduction. We also have proved, through an equivalence with the super sequent calculus, that we only need the newly computed rules to show sequents consisting only of atomic propositions. This is a general result which shows how superdeduction can be used as a logical framework by encapsulating the propositions of the system we want to encode into $\varepsilon$ constructs. Having encoded all reasonable PTS proves how powerful this can be. We also obtain sequent calculus presentations of PTS, which are to be compared to existing ones [16, 19].

We can then use this encoding to check proofs produced by proof assistants based on PTS. To do so, we could, because of the equivalence with first-order logic without modulo but within a compatible theory $\mathcal{T}$, use an intuitionistic first-order theorem prover. To prove a theorem $A$, we have to prove that it is inhabited if seen as a type, and thus to prove $\mathcal{T} \vdash \exists t$. $\varepsilon(t,|A|)$. Notwithstanding, this would probably be very inefficient, because the theorem prover would not be adapted to this particular theory. It should be better to use theorem provers based on supernatural deduction, such as the proof assistant Lemuridæ which has to be adapted for intuitionistic logic. (For the moment, it can be used because classical super rules subsumes intuitionistic ones, but we cannot guarantee the conservativeness.) Possibly, we could also use tools based on deduction modulo- there exists translations between supernatural deduction and deduction modulo that relatively preserve proof structures [7]using procedures such as ENAR [13] or TaMed [6]. But to fully be able to check proofs from assistants, we also need to encode inductive types, that are present in many provers, such as e.g. Coq. This can also naturally be done in superdeduction, as shown by a recent work of Allali, Brauner and Dowek [2].

An important remaining issue is the decidability of type checking/proof search. Type checking is undecidable for PTS in general, but it is decidable for functional PTS that weakly normalizes [5]. In that case, the conservativeness proves that proof search in $\mathrm{NJ}^{+}$is at most semi-decidable, even if we are working modulo an undecidable congruence $\left(\lambda_{W}\right)$. (Note that proof search for atomic sequents with wellformed context in $\mathrm{NJ}^{+}$corresponds to type checking in the PTS.) We conjecture that it is in fact decidable for atomic sequents, due to the strong correspondence between the two systems.

In a related topic, we also have to look at the normaliza- tion of the $\mathrm{NJ}^{+}$system for PTS, i.e. to check if proof terms associated with a $\mathrm{NJ}^{+}$proof (which are in fact $\rho$-terms) strongly normalize. Due to the conservativeness, there is a correspondence between a $\mathrm{NJ}^{+}$proof and a term of the PTS, at least when contexts are well formed. The normalization is therefore probably equivalent to the normalization of the PTS. It remains to extend the rewrite systems with some rules to check for the wellformedness of contexts, to have a full correspondence.

We should also try to encode more deductive systems into superdeduction. Indeed, the naturalness of our translation into supernatural deduction probably comes from the fact that PTS are extensions of simply-typed $\lambda$-calculus which is isomorph to natural deduction. This moderates our claim that superdeduction is a powerful logical framework, although it can encode HOL, (higher-order) arithmetics, Zermelo's set theory and PTS.

\section{References}

[1] M. Abadi, L. Cardelli, P.-L. Curien, and J.-J. Lévy. Explicit substitutions. Journal of Functional Programming, 1(4):375-416, 1991.

[2] L. Allali, P. Brauner, and G. Dowek. A semantic proof of system T termination. Work in progress, 2008.

[3] F. Baader and T. Nipkow. Term Rewriting and all That. Cambridge University Press, 1998.

[4] H. Barendregt. Handbook of logic in computer science, volume 2, chapter Lambda calculi with types, pages 117-309. Oxford University Press, 1992.

[5] G. Barthe. The semi-full closure of pure type systems. In Mathematical Foundations of Computer Science, volume 1450 of $L N C S$, pages 316-325. Springer, 1998.

[6] R. Bonichon and O. Hermant. A semantic completeness proof for TaMeD. In M. Hermann and A. Voronkov, editors, $L P A R$, volume 4246 of $L N C S$, pages 167-181. Springer, 2006.

[7] P. Brauner, G. Dowek, and B. Wack. Normalization in supernatural deduction and in deduction modulo. Submitted, 2007.

[8] P. Brauner, C. Houtmann, and C. Kirchner. Principle of superdeduction. In L. Ong, editor, Proceedings of LICS, pages 41-50, jul 2007.

[9] D. Cousineau and G. Dowek. Embedding pure type systems in the lambda-pi-calculus modulo. In S. Ronchi Della Rocca, editor, TLCA, volume 4583 of $L N C S$, pages 102-117. Springer, 2007.

[10] G. Dowek. Confluence as a cut elimination property. In R. Nieuwenhuis, editor, RTA, volume 2706 of LNCS, pages 2-13. Springer, 2003.

[11] G. Dowek, T. Hardin, and C. Kirchner. Higher-order unification via explicit substitutions. Information and Computation, 157(1/2):183-235, 2000.

[12] G. Dowek, T. Hardin, and C. Kirchner. HOL- $\lambda \sigma$ an intentional first-order expression of higher-order logic. Mathematical Structures in Computer Science, 11(1):1-25, 2001. 
[13] G. Dowek, T. Hardin, and C. Kirchner. Theorem proving modulo. Journal of Automated Reasoning, 31(1):33-72, 2003.

[14] G. Gentzen. Untersuchungen über das logische Schliessen. Mathematische Zeitschrift, 39:176-210, 405-431, 1934. Translated in Szabo, editor., The Collected Papers of Gerhard Gentzen as "Investigations into Logical Deduction".

[15] H. Geuvers. The Church-Rosser property for beta-etareduction in typed lambda-calculi. In LICS, pages 453-460. IEEE Computer Society, 1992.

[16] F. Gutiérrez and B. C. Ruiz. Cut elimination in a class of sequent calculi for pure type systems. Electronic Notes in Theoretical Computer Science, 84, 2003.

[17] R. Harper, F. Honsell, and G. Plotkin. A framework for defining logics. Journal of the ACM, 40(1):143-184, 1993.

[18] D. Kesner. Confluence of extensional and non-extensional $\lambda$-calculi with explicit substitutions. Theoretical Computer Science, 238(1-2):183-220, 2000.

[19] S. Lengrand, R. Dyckhoff, and J. McKinna. A sequent calculus for Pure Type Systems. In Z. Esik, editor, CSL, volume 4207 of LNCS, pages 441-455. Springer, September 2006.

[20] F. Pfenning. The practice of logical frameworks. In $C A A P$, volume 1059 of $L N C S$, pages 119-134. Springer, 1996.

[21] C. Sacerdoti Coen and F. Kirchner. Fellowship, 2006. http://www.lix.polytechnique.fr/Labo/Florent. Kirchner/fellowship/.

[22] A. Waaler and L. Wallen. Handbook of Tableau Methods, chapter Tableaux for Intutionistic Logics, pages 255-296. Kluwer Academic Publishers, Boston, 1999.

[23] B. Wack. Supernatural deduction. Manuscript, available at http://www. loria.fr/ wack/papers/supernatural. ps.gz, 2006. 


\section{Contents}

1. Introduction

2. Prerequisites

2.1. Intuitionistic sequent calculus . . . . . . . 3

2.2. Pure Type Systems . . . . . . . . . . . . . . 3

2.3. A scheme for $\lambda$-calculi with explicit substitutions ............. 3

\section{Superdeductive systems for intuitionistic logic 4}

3.1. Supernatural deduction . . . . . . . . . . . 4

3.2. Super sequent calculus . . . . . . . . . . . 5

3.3. Translations between these systems . . . . 5

4. PTS as superdeductive theories 6

4.1. The system . . . . . . . . . . . . 6

4.2. Soundness and conservativeness . . . . . 7

\section{Applications}

5.1. Using $\lambda_{\sigma}$ for the explicit-substitution calculus 5.2. A sequent calculus for Pure Types Systems .

\section{Conclusion}

A Proofs of the equivalence between $\mathrm{LB}^{+}$and $\mathrm{NJ}^{+} \quad 12$

A.1. Properties of $\mathrm{LB}^{+}$. . . . . . . . . . . . . 12

A.2 Translations from $\mathrm{NJ}^{+}$to $\mathrm{LB}^{+} \ldots . . . . . .14$

A.3. Translations from $\mathrm{LB}^{+}$to $\mathrm{NJ}^{+}$. . . . . . . 16

\section{B. Interactions PTS terms and first-order terms}

C. Why are several $\dot{\pi}$ necessary?

\section{A. Proofs of the equivalence between $\mathrm{LB}^{+}$and $\mathrm{NJ}^{+}$}

\section{A.1. Properties of $\mathrm{LB}^{+}$}

Definition 7 (Proof skeleton). The skeleton of a proof is the tree of the inference rules used in it.

Definition 8 (Structural inclusion). We say that a proof $\pi$ is structurally included into a proof $\Phi$ if the skeleton of $\pi$ is included in the skeleton of $\Phi$ in the sense of tree inclusion.

Proposition 18 (Admissibility of the weakening). If $\Gamma \vdash \Delta$ can be proved in $\mathrm{LB}^{+}$, so does $\Gamma^{\prime}, \Gamma \vdash \Delta, \Delta^{\prime}$, with the same skeleton.

Proof. By induction on the proof and on the formulæ in the right-hand side of the rewrite system.

Lemma 19. Let $R$ be the rewrite rule $A \rightarrow P$ with associated left super rule

$$
R_{L} \frac{\left(\Gamma, \Gamma_{i} \vdash \Delta_{i}, \Delta\right)_{i}}{\Gamma, A \vdash \Delta}
$$

Suppose that for all $i$ there exists some $C_{i} \in \Delta_{i}, \Delta$ such that $\Gamma, \Gamma_{i} \vdash C_{i}$ is provable.

Then there exists some $C \in \Delta$ such that either $\Gamma \vdash C$ is provable or $\Gamma, \Gamma_{i} \vdash \Delta_{i}, C$ is provable for all $i$. Note that in both cases, $\Gamma, A \vdash C$ is provable.

Proof. By induction on $P$ we can easily show that right rules are of the form

$$
R_{L} \frac{\left(\Gamma, \Gamma_{i} \vdash \Delta_{i}, \Delta\right)_{i \in I}}{\Gamma, A \vdash \Delta}
$$

and that there exists $i_{0} \in I$ such that for all $i \in I, \Gamma_{i_{0}} \subseteq \Gamma_{i}$.

We proceed by induction on $P$.

Suppose that for all $i$ there exists some $C_{i} \in \Delta_{i}, \Delta$ such that $\Gamma, \Gamma_{i} \vdash C_{i}$ is provable.

If $C_{i_{0}} \in \Delta$, then $\Gamma, \Gamma_{i_{0}} \vdash C_{i_{0}}$ is provable, and by weakening, for all $i$ we can prove $\Gamma, \Gamma_{i} \vdash \Delta_{i}, C_{i_{0}}$.

If $C_{i_{0}} \in \Delta_{i_{0}}$, we can show that for all $i$, if $C \in \Delta_{i}$ then $C$ appears on the left of some implication in $P$. Therefore $P=C\left[C_{i_{0}} \Rightarrow P^{\prime}\right]$ for some context $C$ and proposition $P^{\prime}$. Let $S$ be the rewrite rule $B \rightarrow C\left[P^{\prime}\right]$. Then by definition, if $I^{\prime}=$ $\left\{i \in I: C_{i_{0}} \notin \Delta_{i}\right\}$, the left rule for $S$ is

$$
S_{L} \frac{\left(\Gamma, \Gamma_{i} \vdash \Delta_{i}, \Delta\right)_{i \in I^{\prime}}}{\Gamma, B \vdash \Delta}
$$

By induction hypothesis, there exists some $C \in \Delta$ such that either $\Gamma \vdash C$ is provable, which permits to conclude, or for all $i \in I^{\prime}, \Gamma, \Gamma_{i} \vdash \Delta_{i}, C$ is provable. It remains to be proved that for all $i \in I \backslash I^{\prime}, \Gamma, \Gamma_{i} \vdash \Delta_{i}, C$, but if $i \in I \backslash I^{\prime}$, then $\Gamma_{i_{0}} \subseteq \Gamma_{i}$ and $C_{i_{0}} \in \Delta_{i}$ so that we can conclude by weakening with the proof of $\Gamma, \Gamma_{i_{0}} \vdash C_{i_{0}}$.

Note: The proof of $\Gamma, A \vdash C$ obtained at the end is structurally included in the proof of $\Gamma, A \vdash \Delta$ we get by applying $R_{L}$ to the proofs of the supposition.

Lemma 20. Let $R$ be the rewrite rule $A \rightarrow P$.

The associated right super rule is of the form

$$
R_{R} \frac{\left(\Gamma, \Gamma_{i} \vdash A_{i}\right)_{i \in I} \quad\left(\Gamma \vdash A_{j}, \Delta\right)_{j \in J}}{\Gamma \vdash A, \Delta}
$$

Suppose for all $i \in I$ we can prove $\Gamma, \Gamma_{i} \vdash A_{i}$ and for all $j \in J$ there exists some $C_{j} \in A_{j}, \Delta$ such that we can prove $\Gamma \vdash C_{j}$.

Then either $\Gamma \vdash A_{j}$ is provable for all $j \in J$ or there exists some $C \in \Delta$ such that $\Gamma \vdash \Delta$. In both cases, there exists some $C \in A, \Delta$ such that $\Gamma \vdash C$ is provable. 
Proof. To prove the form of the right rule, we proceed by induction on $P$. The only difficult case is the implication: suppose $P=P_{1} \Rightarrow P_{2}$. Let $S$ be the rewrite rule $B \rightarrow P_{2}$. By induction hypothesis, the right rule for $S$ is of the form

$$
S_{R} \frac{\left(\Gamma, \Gamma_{i} \vdash A_{i}\right)_{i \in I} \quad\left(\Gamma \vdash A_{j}, \Delta\right)_{j \in J}}{\Gamma \vdash B, \Delta}
$$

Then by definition the right rule for $R$ is

$$
S_{R} \frac{\left(\Gamma, P_{1}, \Gamma_{i} \vdash A_{i}\right)_{i \in I} \quad\left(\Gamma, P_{1} \vdash A_{j}\right)_{j \in J}}{\Gamma \vdash A, \Delta}
$$

which is of the convenient form.

To prove the rest of the lemma, suppose for all $i \in I$ we can prove $\Gamma, \Gamma_{i} \vdash A_{i}$ and for all $j \in J$ there exists some $C_{j} \in A_{j}, \Delta$ such that we can prove $\Gamma \vdash C_{j}$. If there exists $j \in J$ such that $C_{j} \in \Delta$, then we conclude because $\Gamma \vdash C_{j}$. Otherwise, for all $j \in J$ we have $C_{j}=A_{j}$. That means that for all $j \in J$ we can prove $\Gamma \vdash A_{j}$ and by applying the right rule for $R$ we get a proof of $\Gamma \vdash A$.

Note: The proof of $\Gamma \vdash C$ obtained at the end is structurally included in the proof of $\Gamma \vdash A, \Delta$ we get by applying $R_{R}$ to the proofs of the supposition.

Proposition 21 (Constructivism). If $\pi$ is a proof of $\Gamma \vdash \Delta$ $\mathrm{LB}^{+}$, then there exists some $C \in \Delta$ and of proof of $\Gamma \vdash C$ in $\mathrm{LB}^{+}$which is structurally included in $\pi$.

Proof. By induction on the proof. If the last rule is an Axiom or a logical rule, we can easily conclude by induction hypothesis.

If it is a Cut

$$
\operatorname{Cut} \frac{\Gamma, P \vdash \Delta \quad \Gamma \vdash P, \Delta}{\Gamma \vdash \Delta}
$$

by induction hypothesis on $\varpi$, there exists some $C \in P, \Delta$ such that there exists a proof $\varpi^{\prime}$ of $\Gamma \vdash C$ in $\mathrm{LB}^{+}$. If $C$ is in $\Delta, \bar{\sigma}^{\prime}$ is a convenient proof. If $C=P$, by weakening $\pi^{\prime}$ is also a proof of $\Gamma \vdash P, C$ By induction hypothesis on $\pi$, there exists some $C \in \Delta$ such that there exists a proof $\pi^{\prime}$ of $\Gamma, P \vdash C$ in $\mathrm{LB}^{+}$. A convenient proof is therefore

$$
\operatorname{Cut} \frac{\Gamma, P \vdash C \quad \Gamma \vdash P, C}{\Gamma \vdash C}
$$

If it is a left super rule, then we can conclude by induction hypothesis and Lemma 19, and if it is a right super rule, by induction hypothesis and Lemma 20.

Lemma 22. If we have a formula $P$ of degree $d$ greater or equal than 2 (i.e. non-atomic), if we have proofs $\pi$ of $\Gamma, P \vdash \Delta^{\prime}$ and $\pi^{\prime}$ of $\Gamma^{\prime} \vdash P, \Delta^{\prime}$ of degree less than $d$, then we can a proof of $\Gamma, \Gamma^{\prime} \vdash \Delta, \Delta^{\prime}$ of degree less than $d$.
Proof. First remark that if a proof is structurally included into another, then its degree is less or equal, and that weakening a proof preserve its degree.

First, let remark that we can consider that $\Delta^{\prime}=\emptyset$. Indeed, by Proposition 21 with $\pi^{\prime}$, we can prove either $\Gamma^{\prime} \vdash P$ or $\Gamma^{\prime} \vdash \Delta^{\prime}$ by a proof of degree less than $d$. In the second case we conclude by weakening.

By induction on $\pi, \pi^{\prime}$.

1. If $\pi$ ends with an axiom. Then either there is some $C \in \Gamma \cap \Delta$ in which case we can prove $\Gamma, \Gamma^{\prime} \vdash \Delta$ with an axiom; or $P \in \Delta$, in which case $\pi^{\prime}$ is by weakening a proof of $\Gamma, \Gamma^{\prime} \vdash \Delta$.

2. If $\pi^{\prime}$ ends with an axiom: dual to 1 .

3. If $\pi$ ends with a logical or super rule with the active formula $A \in \Gamma \cup \Delta$. If $A \in \Gamma$, let $\Gamma^{\prime \prime}$ be $\Gamma \backslash\{A\}$ and $\Delta^{\prime \prime}$ be $\Delta$, otherwise let $\Gamma^{\prime \prime}$ be $\Gamma$ and $\Delta^{\prime \prime}$ be $\Delta \backslash\{A\}$. $\pi$ is

$$
\mathrm{r} \frac{\left(\Gamma^{\prime \prime}, P, \Gamma_{i} \vdash \Delta_{i}, \Delta^{\prime \prime}\right)_{i} \quad\left(\Gamma^{\prime \prime}, P, \Gamma_{j} \vdash \Delta_{j}\right)_{j}}{\Gamma, P \vdash \Delta}
$$

By induction hypothesis, there exists proofs $\varpi_{i}$ of degree less than $d$ of $\Gamma^{\prime \prime}, \Gamma^{\prime}, \Gamma_{i} \vdash \Delta_{i}, \Delta^{\prime \prime}$ and proofs $\varpi_{j}$ of $\Gamma^{\prime \prime}, \Gamma^{\prime}, \Gamma_{j} \vdash \Delta_{j}$.

We conclude with

$$
\mathrm{r} \frac{\left(\Gamma^{\prime \prime}, \Gamma^{\prime}, \Gamma_{i} \vdash \Delta_{i}, \Delta^{\prime \prime}\right)_{i} \quad\left(\Gamma^{\prime \prime}, \Gamma^{\prime}, \Gamma_{j} \vdash \Delta_{j}\right)_{j}}{\Gamma, \Gamma^{\prime} \vdash \Delta}
$$

4. If $\pi^{\prime}$ ends with a logical or super rule with the active formula $A \in \Gamma^{\prime}$. Let $\Sigma$ be $\Gamma^{\prime} \backslash\{A\}$. $\pi^{\prime}$ is

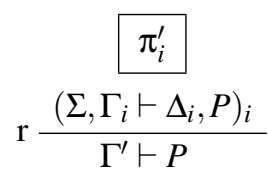

By induction hypothesis, there exists proofs $\varpi_{i}^{\prime}$ of degree less than $d$ of $\Gamma, \Sigma, \Gamma_{i} \vdash \Delta_{i}, \Delta$.

We conclude with

$$
\mathrm{r} \frac{\left(\Gamma, \Sigma, \Gamma_{i} \vdash \Delta_{i}^{\prime}, \Delta\right)_{i}}{\Gamma, \Gamma^{\prime} \vdash \Delta}
$$

5. If both $\pi$ and $\pi^{\prime}$ end with logical or super rules with $P$ as active formula. Because $P$ is not atomic, these rules are in fact logical rules. 
- If $P=Q \wedge R, \pi$ is

$$
\wedge_{L} \frac{\Gamma, Q, R \vdash \Delta}{\Gamma, Q \wedge R \vdash \Delta}
$$

and $\pi^{\prime}$

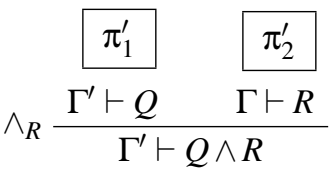

We conclude with

$$
\begin{aligned}
& \begin{array}{rrr}
\pi_{1} & \pi_{2}^{\prime} & \Gamma, \Gamma^{\prime}, Q, R \vdash \Delta \\
\hline, \Gamma^{\prime}, Q \vdash R, \Delta & \pi_{1}^{\prime} \\
\hline
\end{array} \\
& \text { Cut } \frac{\Gamma, \Gamma^{\prime}, Q \vdash \Delta}{\Gamma, \Gamma^{\prime} \vdash \Delta} \quad \Gamma, \Gamma^{\prime} \vdash Q, \Delta
\end{aligned}
$$

- If $P$ is $Q \Rightarrow R, \pi$ is

$$
\Rightarrow_{L} \frac{\Gamma \vdash Q, \Delta \quad \Gamma, R \vdash \Delta}{\pi_{1}}
$$

and $\pi^{\prime}$ is

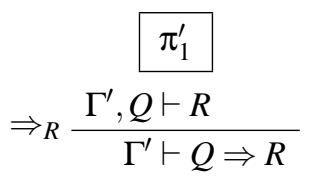

We conclude with

$$
\operatorname{Cut} \frac{\Gamma_{,} \Gamma^{\prime}, Q, R \vdash \Delta \quad \Gamma, \Gamma^{\prime}, Q \vdash R, \Delta}{\operatorname{Cut} \frac{\Gamma, \Gamma^{\prime}, Q \vdash \Delta}{\pi_{1}^{\prime}}} \underset{\Gamma, \Gamma^{\prime} \vdash Q, \Delta}{\pi_{1}}
$$

- If $P$ is $\forall x . Q$, then $\pi$ is

$$
\forall_{L} \frac{\Gamma,\{t / x\} Q \vdash \Delta}{\Gamma, \forall x \cdot Q \vdash \Delta}
$$

for some $t$ and $\pi^{\prime}$

$$
\forall_{R} \frac{\Gamma_{1}^{\prime} \vdash Q}{\Gamma^{\prime} \vdash \forall x . Q} x \text { not free in } \Gamma^{\prime}
$$

As $x$ is free in $\Gamma^{\prime}$ we can replace it by $t$ in $\pi_{1}^{\prime}$ to get a proof $\varpi_{1}^{\prime}$ of $\Gamma^{\prime} \vdash\{t / x\} Q$ of the same degree.

We conclude with

$$
\operatorname{Cut} \frac{\Gamma,, \Gamma^{\prime}\{t / x\} Q \vdash \Delta}{\pi_{1}} \begin{gathered}
\varpi_{1}^{\prime} \\
\Gamma, \Gamma^{\prime} \vdash \Delta
\end{gathered}
$$

Proposition 6 (Atomic $\mathrm{LB}^{+}$). If the rewrite system is ppf, and $\Gamma$ and $\Delta$ contains only atomic formula, then $\Gamma \vdash \Delta$ is provable iff it is provable with only identity and super rules.

Proof. First remark that the rewrite system is ppf iff the super rules of $\mathrm{LB}^{+}$decompose atomic formuæ into atomic formulæ.

Suppose there is a proof of $\Gamma \vdash \Delta$. By induction on this proof. As nor $\Gamma$ neither $\Delta$ contain non atomic formulæ, there cannot be a logical rule at the root of the proof. If the rule at the root of the proof is an Axiom, we are done. If it is a super rule, we can conclude with the remark above by induction hypothesis. If it is a Cut, it remains to be proved that we only need Cuts around atomic formulæ.

Note that as Cut may not be admissible (see below), we cannot avoid having Cuts in our proofs. Nevertheless, we can use some kind of cut elimination procedure to reduce to the case of atomic Cut.

Let suppose the last rule is a Cut, we proceed by induction on the degree of $P$ :

$$
\operatorname{Cut} \frac{\Gamma, P \vdash \Delta}{\pi} \quad \begin{array}{cc}
\pi & \Gamma \\
\Gamma \vdash \Delta & \Gamma
\end{array}
$$

If the degree $d$ of $P$ is greater than 1, we can apply the induction hypothesis on the subproof and Lemma 22 to get a proof of $\Gamma, \Gamma \vdash \Delta, \Delta$ of degree less than $d$. Because $\Gamma$ and $\Delta$ contains only atomic formulæ, we can use Cut to simulate contraction:

$$
\text { Cut } \frac{\Gamma, A, A \vdash \Delta \quad \text { Axiom } \overline{\Gamma, A \vdash A, \Delta}}{\Gamma, A \vdash \Delta}
$$

The proof we obtain is therefore if degree less than the degree of $P$, so we can apply the induction hypothese to conclude.

\section{A.2. Translations from $\mathrm{NJ}^{+}$to $\mathrm{LB}^{+}$}

For convenience, proofs in $\mathrm{NJ}^{+}$in this section and the following will be represented a la Gentzen [14], instead of in sequent style.

Lemma 23. Let $R$ be the rewrite rule $A \rightarrow P$. An elimination rule for $R$ is of the form

$$
R_{E p} \frac{A \quad H_{1} \quad \cdots \quad H_{n}}{K}
$$


A left rule for $R$ is of the form

$$
R_{L} \frac{\left(\Gamma, \Gamma_{i} \vdash \Delta_{i}, \Delta\right)_{i}}{\Gamma, A \vdash \Delta}
$$

Suppose we have proofs of $\Gamma \vdash H_{j}$ in $\mathrm{LB}^{+}$for all $j$.

Then we can prove $\Gamma, \Gamma_{i} \vdash \Delta_{i}, K$ in $\mathrm{LB}^{+}$(and thus also $\Gamma, A \vdash K)$.

Proof. The form of the elimination and left rules can be shown by a simple induction.

The remaining can be proved by induction on the formula $P$.

- Suppose $P=B$ is atomic.

Then the elimination rule of $R$ is

$$
R_{E} \frac{A}{B}
$$

The left rule for $R$ is

$$
R_{L} \frac{\Gamma, B \vdash \Delta}{\Gamma, A \vdash \Delta}
$$

We have

$$
\text { Axiom } \overline{\Gamma, B \vdash B}
$$

- Suppose $P=\perp$.

Then the elimination rule of $R$ is

$$
R_{E} \frac{A}{C}
$$

for all $C$.

The left rule for $R$ is

$$
R_{L} \overline{\Gamma, A \vdash \Delta}
$$

As it has no premises, nothing has to be proven. Notwithstanding, remark that $\Gamma, A \vdash C$ is easily provable.

- Suppose $P$ is $P_{1} \Rightarrow P_{2}$.

Let $S$ be the rewrite rule $D \rightarrow P_{2}$.

An elimination rule for $S$ is of the form

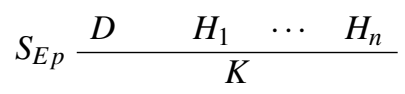

and elimination rules for $R$ are then

$$
R_{E p} \begin{array}{ccccc}
A & P_{1} & H_{1} & \cdots & H_{n} \\
\hline \multicolumn{7}{c}{K}
\end{array}
$$

The left rule for $S$ is of the form

$$
S_{L} \frac{\left(\Gamma, \Gamma_{i} \vdash \Delta_{i}, \Delta\right)_{i}}{\Gamma, D \vdash \Delta}
$$

and then the left rule for $R$ is

$$
R_{L} \frac{\left(\Gamma, \Gamma_{i} \vdash \Delta_{i}, \Delta\right)_{i} \quad \Gamma \vdash P_{1}, \Delta}{\Gamma, A \vdash \Delta}
$$

Suppose we have proofs $\pi_{j}$ of $\Gamma \vdash H_{j}$ and a proof $\pi_{0}$ of $\Gamma \vdash P_{1}$.

By induction hypothesis, there are proofs of $\Gamma, \Gamma_{i} \vdash$ $\Delta_{i}, K$ for all $i$, and by weakening $\pi_{0}$ is a proof of $\Gamma \vdash P_{1}, K$.

Note: This could not be done in $\mathrm{LJ}^{+}$, because we cannot have multiple formulæ in the right part of a sequent.

- Suppose $P$ is $P_{1} \wedge P_{2}$.

Let $R_{1}$ be the rewrite rule $A_{1} \rightarrow P_{1}$ and $R_{2}$ be the rewrite rule $A_{2} \rightarrow P_{2}$.

For $k \in\{1 ; 2\}$, an elimination rule for $R_{k}$ is of the form

$$
R_{k E p} \frac{A_{k} \quad H_{k, p}^{1} \quad \cdots \quad H_{k, p}^{n}}{K_{k, p}}
$$

and elimination rules for $R$ are then

$$
R_{E k, p} \frac{A \quad H_{k, p}^{1} \quad \cdots \quad H_{k, p}^{n}}{K_{k, p}}
$$

The left rule for $R_{k}$ is of the form

$$
R_{k L} \frac{\left(\Gamma, \Gamma_{i} \vdash \Delta_{i}, \Delta\right)_{i \in I_{k}}}{\Gamma, A_{k} \vdash \Delta}
$$

and then the left rule for $R$ is

$$
R_{L} \frac{\left(\Gamma, \Gamma_{i}, \Gamma_{j} \vdash \Delta_{i}, \Delta_{j}, \Delta\right)_{i \in I_{1}, j \in I_{2}}}{\Gamma, A \vdash \Delta}
$$

Let $k$ be 1 or 2 .

Suppose we have proofs $\pi_{j}$ of $\Gamma \vdash H_{k, p}^{j}$.

By induction hypothesis with $R_{k}$, there are proofs of $\Gamma, \Gamma_{i} \vdash \Delta_{i}, K_{k, p}$ for all $p$ and $i \in I_{k}$. By weakening, these are also proofs of $\Gamma, \Gamma_{i}, \Gamma_{j} \vdash \Delta_{i}, \Delta_{j}, K_{k, p}$ for all $j \in I_{3-k}$.

- Suppose $P$ is $\forall x$. $Q$. Let $t$ be a term actually substituted for $x$ in $R_{L}$.

Let $S$ be the rewrite rule $D \rightarrow\{t / x\} Q$.

An elimination rule for $S$ is of the form 


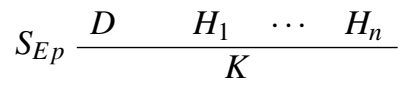

and elimination rules for $R$ are then

$$
R_{E p} \frac{A \quad H_{1} \quad \cdots \quad H_{n}}{K}
$$

The left rule for $S$ is of the form

$$
S_{L} \frac{\left(\Gamma, \Gamma_{i} \vdash \Delta_{i}, \Delta\right)_{i}}{\Gamma,\{t / x\} Q \vdash \Delta}
$$

and then the left rule for $R$ with $t$ is

$$
S_{L} \frac{\left(\Gamma, B, \Gamma_{i} \vdash \Delta_{i}, \Delta\right)_{i}}{\Gamma, A \vdash \Delta}
$$

Suppose we have proofs $\pi_{j}$ of $\Gamma \vdash H_{j}$.

By induction hypothesis, there are proofs of $\Gamma, \Gamma_{i} \vdash$ $\Delta_{i}, K$ for all $i$.

\section{THEOREM 7.}

A proof of C from $\Gamma$ in $\mathrm{NJ}^{+}$can be translated into a proof of $\Gamma \vdash C$ in $\mathrm{LB}^{+}$.

Proof. By induction on the $\mathrm{NJ}^{+}$proof:

- If the last inference rule is a logical rule, then we can use the same translation as [14].

- If the last rule is a super introduction rule, it is easy to see that super introduction rules for $\mathrm{NJ}^{+}$are subcases of super right rules for $\mathrm{LB}^{+}$with only one formula in the right part of the sequents. We can therefore conclude by induction.

- If the last rule is a super elimination rule of the form

$$
R_{E p} \frac{A \quad H_{1} \quad \cdots \quad H_{n}}{C}
$$

By induction hypothesis, there is a $\mathrm{LB}^{+}$proof $\pi_{0}$ of $\Gamma \vdash A$ and there are $\mathrm{LB}^{+}$proofs $\pi_{j}$ of $\Gamma \vdash H_{j}$ for all $j$.

By Lemma 23, there is a proof $\varpi$ of $\Gamma, A \vdash C$ in $\mathrm{LB}^{+}$.

By weakening, $\pi_{0}$ is also a proof of $\Gamma \vdash A, C$.

We can conclude with

$$
\operatorname{Cut} \frac{\Gamma, A \vdash C \quad \Gamma \vdash A, C}{\Gamma} \frac{\pi}{\pi_{0}}
$$

\section{A.3. Translations from $\mathrm{LB}^{+}$to $\mathrm{NJ}^{+}$}

Lemma 24. Suppose we have a $\mathrm{LJ}^{+}$proof whose last inference is

$$
R_{L} \frac{\left(\Gamma_{i} \vdash \Delta_{i}\right)_{i}}{\Gamma, A \vdash \Delta}
$$

where $R$ is $A \rightarrow P$. Suppose for all $i$ there is some $C_{i}$ in $\Delta_{i}$ such that we have supernatural deduction proofs $\pi_{i}$ of $C_{i}$ from assumptions $\Gamma_{i}$.

Then there is some $C$ in $\Delta$ such that it is possible to build a supernatural deduction proof of $C$ from assumptions $\Gamma, A$ and using only elimination rules of $R$ and the proofs $\pi_{i}$.

Proof. By induction on the formula $P$.

- Suppose $P=B$ is atomic.

Then the last rule of the sequent calculus proof is in fact

$$
R_{L} \frac{\Gamma, B \vdash \Delta}{\Gamma, A \vdash \Delta}
$$

Therefore, if $C \in \Delta$ and $\pi$ is a proof of $C$ from $\Gamma$ and $B$, then

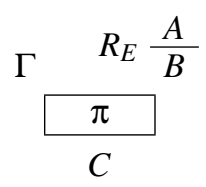

is the proof we want.

- Suppose $P=\perp$. Then the last rule of the sequent calculus proof is in fact

$$
R_{L} \overline{\Gamma, A \vdash C}
$$

Then

$$
R_{E} \frac{A}{C}
$$

is the proof we want.

- Suppose $P$ is $P_{1} \Rightarrow P_{2}$.

Let $S$ be the rewrite rule $D \rightarrow P_{2}$. By definition of the left rules, there exists some $i_{0}$ such that $\left(\Gamma_{i_{0}} \vdash \Delta_{i_{0}}\right)=$ $\left(\Gamma \vdash P_{1}, \Delta\right)$ and

$$
S_{L} \frac{\left(\Gamma_{i} \vdash \Delta_{i}\right)_{i \neq i_{0}}}{\Gamma, D \vdash \Delta}
$$


Suppose that for all $i \neq i_{0}$ we have proofs $\pi_{i}$ from $\Gamma_{i}$ to $C_{i}$ for some $C_{i} \in \Delta_{i}$. By induction hypothesis, we can build a proof $\sigma$ of $C$ from $\Gamma, D, \pi_{i}$ for $i \neq i_{0}$, and elimination rules for $S$.

An elimination rule for $S$ is of the form

$$
S_{E p} \frac{D \quad H_{1} \quad \cdots \quad H_{n}}{K}
$$

and elimination rules for $R$ are then

$$
R_{E p} \begin{array}{ccccc}
A & P_{1} & H_{1} & \cdots & H_{n} \\
\hline K &
\end{array}
$$

By replacing $S_{E p}$ by $R_{E p}$ in $\varpi$ we therefore obtain a proof of $C$ from $\Gamma, A, B$, proofs $\pi_{i}$ for $i \neq i_{0}$ and elimination rules for $R$.

Suppose that there is some $C \in P_{1}, \Delta$ such that we have a proof $\pi_{i_{0}}$ from $\Gamma$ to $C$ (or equivalently from $\Gamma_{i_{0}}$ to $C_{i_{0}} \in \Delta_{i_{0}}$ ). There are two cases : Either $C$ is in $\Delta$, and thus $\pi_{i_{0}}$ is a convenient proof. Or $C=P_{1}$, and then we can build a proof from $\Gamma, A$, proofs $p_{i}$ for all $i$ and elimination rules for $R$ by replacing the assumption $P_{1}$ in $\varpi$ by $\pi_{i_{0}}$.

- Suppose $P$ is $P_{1} \wedge P_{2}$.

Let $R_{1}$ be the rewrite rule $A_{1} \rightarrow P_{1}$ and $R_{2}$ be the rewrite rule $A_{2} \rightarrow P_{2}$.

The left rule for $R_{k}$ is of the form

$$
R_{k L} \frac{\left(\Gamma, \Gamma_{i} \vdash \Delta_{i}, \Delta\right)_{i \in I_{k}}}{\Gamma, D \vdash \Delta}
$$

and then the left rule for $R$ is

$$
R_{L} \frac{\left(\Gamma, \Gamma_{i}, \Gamma_{j} \vdash \Delta_{i}, \Delta_{j}, \Delta\right)_{i \in I_{1}, j \in I_{2}}}{\Gamma, A \vdash \Delta}
$$

Suppose that for all $i \in I_{1}, j \in I_{2}$ we have proofs $\pi_{i, j}$ from $\Gamma, \Gamma_{i}, \Gamma_{j}$ to $C_{i}$ for some $C_{i} \in \Delta_{i}, \Delta_{j}, \Delta$. By induction hypothesis for $R_{1}$, there exists some $C_{j} \in \Delta_{j}, \Delta$ such that there is a proof $\varpi_{j}$ of $C_{j}$ from assumptions $\Gamma$, $\Gamma_{j}, A_{1}$, proof $\pi_{i, j}$ and elimination rules for $R_{1}$.

An elimination rule for $R_{1}$ is of the form

$$
R_{1 E p} \frac{A_{1} \quad H_{1} \quad \cdots \quad H_{n}}{K}
$$

and some elimination rules for $R$ are then

$$
R_{E 1, p} \frac{A \quad H_{1} \quad \cdots \quad H_{n}}{K}
$$

By replacing $R_{1 E p}$ by $R_{E 1, p}$ in $\bar{\varpi}_{j}$ we therefore obtain a proof $\varpi_{j}^{\prime}$ of $C_{j}$ from $\Gamma, \Gamma_{j}, A$, proofs $\pi_{i, j}$ and elimination rules for $R$.

By induction hypothesis for $R_{2}$ with these $\bar{\varpi}_{j}^{\prime}$, there exists some $C \in \Delta$ such that there is a proof $\varpi$ of $C$ from assumptions $\Gamma, A_{2}$, proof $\varpi_{j}^{\prime}$ and elimination rules for $R_{2}$. Once again, if we replace $R_{2 E p}$ by $R_{E 2, p}$ in $\varpi$ we obtain a proof of $C$ from $\Gamma, A$, proofs $\bar{\sigma}_{j}^{\prime}$ and elimination rules for $R$. But proofs $\varpi_{j}^{\prime}$ use only proofs $\pi_{i, j}$ and elimination rules for $R$, so this proof is convenient.

- Suppose $P$ is $\forall x$. $Q$. Let $t$ be the term actually substituted for $x$ in the super sequent calculus proof of $\Gamma, A \vdash \Delta$.

Let $S$ be the rewrite rule $D \rightarrow\{t / x\} Q$. By definition of the left rules, we have

$$
S_{L} \frac{\left(\Gamma_{i} \vdash \Delta_{i}\right)_{i}}{\Gamma, D \vdash \Delta}
$$

Suppose that for all $i$ we have proofs $\pi_{i}$ from $\Gamma_{i}$ to $C_{i}$ for some $C_{i} \in \Delta_{i}$. By induction hypothesis, there exists some $C \in \Delta$ such that we can build a proof $\varpi$ of $C$ from $\Gamma, D$, proofs from $\pi_{i}$ and elimination rules for $S$.

An elimination rule for $S$ is of the form

$$
S_{E p} \frac{D \quad H_{1} \quad \cdots \quad H_{n}}{K}
$$

and elimination rules for $R$ are then

$$
R_{E p} \frac{A \quad H_{1} \quad \cdots \quad H_{n}}{K}
$$

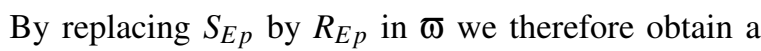
proof of $C$ from $\Gamma, A$, proofs $\pi_{i}$ and elimination rules for $R$.

Lemma 25. Suppose we have a $\mathrm{LJ}^{+}$proof whose last inference is

$$
R_{R} \frac{\left(\Gamma_{i} \vdash \Delta_{i}\right)_{i}}{\Gamma \vdash A, \Delta}
$$

where $R$ is $A \rightarrow P$. Suppose for all $i$ there is some $C_{i}$ in $\Delta_{i}$ such that we have supernatural deduction proofs $\pi_{i}$ of $C_{i}$ from assumptions $\Gamma_{i}$.

Then there is some $C$ in $A, \Delta$ such that it is possible to build a supernatural deduction proof of $C$ from assumptions $\Gamma$ and using only the proofs $\pi_{i}$, with the introduction rules of $R$ at the root if $C=A$.

Proof. By induction on the formula $P$. 
- Suppose $P=B$ is atomic.

Then the last rule of the sequent calculus proof is in fact

$$
R_{R} \frac{\Gamma \vdash B, \Delta}{\Gamma \vdash A, \Delta}
$$

Suppose we have a proof $\pi$ of $B$ from $\Gamma$. Then

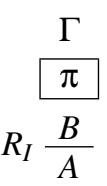

is a convenient proof. Else, if we have a proof $\pi$ of some other $C \in \Delta$ from $\Gamma$, this is a convenient proof.

- Suppose $P=\perp$. Then the last rule of the sequent calculus proof is in fact

$$
R_{R} \frac{\Gamma \vdash \perp, \Delta}{\Gamma \vdash A, \Delta}
$$

Suppose we have a proof $\pi$ of $\perp$ from $\Gamma$. Then

$$
\begin{array}{r|}
\multicolumn{1}{c}{\Gamma} \\
R_{I} \frac{\perp}{A} \\
\hline
\end{array}
$$

is a convenient proof. Else, if we have a proof $\pi$ of some other $C \in \Delta$ from $\Gamma$, this is a convenient proof.

- Suppose $P$ is $P_{1} \Rightarrow P_{2}$.

Let $S$ be the rewrite rule $D \rightarrow P_{2}$. By definition of the right rules,

$$
S_{R} \frac{\left(\Gamma_{i} \vdash \Delta_{i}\right)_{i}}{\Gamma, P_{1} \vdash D}
$$

(Remark there is only $D$ in the right part of the conclusion.)

Suppose that for all $i$ we have proofs $\pi_{i}$ from $\Gamma_{i}$ to $C_{i}$ for some $C_{i} \in \Delta_{i}$. By induction hypothesis, we can build a proof $\Phi$ of $D$ from $\Gamma, P_{1}$, proofs $\pi_{i}$ and the introduction rule for $S$.

The introduction rule for $S$ is of the form

$$
\begin{array}{cc}
{\left[A_{1}^{1}, \cdots, A_{1}^{p_{1}}\right]} & {\left[A_{m}^{1}, \cdots, A_{m}^{p_{m}}\right]} \\
S_{I} \frac{B_{1}}{2} & D
\end{array}
$$

and the introduction rule for $R$ is then

$$
\begin{array}{cc}
{\left[P_{1}, A_{1}^{1}, \cdots, A_{1}^{p_{1}}\right]} & {\left[P_{1}, A_{m}^{1}, \cdots, A_{m}^{p_{m}}\right]} \\
R_{I} \frac{B_{1}}{A} & B_{m} \\
A
\end{array}
$$

By replacing $S_{I}$ by $R_{I}$ at the root of $\varpi$ we therefore obtain a proof of $A$ from $\Gamma$, proofs $\pi_{i}$ and the introduction rule for $R$ at the root. ( $P_{1}$ is in the assumptions of all premises of $R_{I}$, so that it is no longer an assumption of the resulting proof.)

Note: Here appears the difference between $\mathrm{LB}^{+}$ and $\mathrm{LK}^{+}$. If we allow multiple right formulæ in the premises of the right rule for $\Rightarrow$, we do not necessarily obtain a proof of $D$ from $\Gamma$ and $P_{1}$, but we can have a proof of some other $C \in \Delta$, and therefore we cannot conclude. This explains why, with the rewrite system $A \rightarrow(B \Rightarrow C) \wedge D$, the sequent $D \vdash A, B$ can be proved in $\mathrm{LK}^{+}$but not in $\mathrm{LB}^{+}$nor either $A$ or $B$ from $D$ in $\mathrm{NJ}^{+}$.

- Suppose $P$ is $P_{1} \wedge P_{2}$.

Let $R_{1}$ be the rewrite rule $A_{1} \rightarrow P_{1}$ and $R_{2}$ be the rewrite rule $A_{2} \rightarrow P_{2}$.

By definition of the right rules, there exists $I_{1}$ and $I_{2}$ such that for $k \in\{1 ; 2\}$

$$
R_{k R} \frac{\left(\Gamma_{i} \vdash \Delta_{i}\right)_{i \in I_{k}}}{\Gamma \vdash A_{k}, \Delta}
$$

and

$$
R_{R} \frac{\left(\Gamma_{i} \vdash \Delta_{i}\right)_{i \in I_{1}} \quad\left(\Gamma_{i} \vdash \Delta_{i}\right)_{i \in I_{2}}}{\Gamma \vdash A, \Delta}
$$

Suppose that for all $i \in I_{1} \cup I_{2}$ we have proofs $\pi_{i}$ from $\Gamma_{i}$ to $C_{i}$ for some $C_{i} \in \Delta_{i}$. By induction hypothesis, there exists some $C_{k} \in A_{k}, \Delta$ such that we can build a proof $\varpi_{k}$ of $C$ from $\Gamma$, proofs $\pi_{i}$ and the introduction rule for $R_{k}$.

If $C_{k} \in \Delta$ for $k=1$ or $k=2, \varpi_{k}$ is a convenient proof. In the other case, the introduction rule for $R_{k}$ is of the form

$$
\begin{gathered}
{\left[A_{k, 1}^{1}, \cdots, A_{k, 1}^{p_{1}}\right]} \\
R_{k I} \frac{\left[A_{k, m}^{1}, \cdots, A_{k, m}^{p_{m}}\right]}{B_{k, 1}}
\end{gathered}
$$

and the introduction rule for $R$ is then

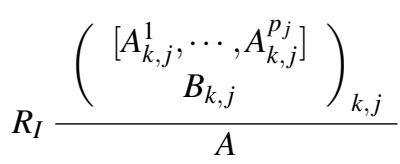


We can replace $R_{1 I}$ and $R_{2 I}$ at the root of $\varpi_{1}$ and $\varpi_{2}$ by $R_{I}$ to obtain a proof of $A$ from $\Gamma$, proofs $\pi_{i}$ and the introduction rule for $R$ at the root.

- Suppose $P$ is $\forall x$. $Q$.

Let $S$ be the rewrite rule $D(x) \rightarrow Q$. Supposing $x$ is free in $\Gamma$, by definition of the right rules,

$$
S_{R} \frac{\left(\Gamma_{i} \vdash \Delta_{i}\right)_{i}}{\Gamma \vdash D(x)}
$$

Suppose that for all $i$ we have proofs $\pi_{i}$ from $\Gamma_{i}$ to $C_{i}$ for some $C_{i} \in \Delta_{i}$. By induction hypothesis, we can build a proof $\varpi$ of $D(x)$ from $\Gamma$, proofs $\pi_{i}$ and the introduction rule for $S$.

The introduction rule for $S$ is of the form

$$
\begin{array}{cc}
{\left[A_{1}^{1}, \cdots, A_{1}^{p_{1}}\right]} & {\left[A_{m}^{1}, \cdots, A_{m}^{p_{m}}\right]} \\
S_{I} \frac{B_{1}}{D(x)} & B_{m}
\end{array}
$$

and the introduction rule for $R$ is then

$$
\begin{array}{cc}
{\left[A_{1}^{1}, \cdots, A_{1}^{p_{1}}\right]} & {\left[A_{m}^{1}, \cdots, A_{m}^{p_{m}}\right]} \\
R_{I} \frac{B_{1}}{B_{m}} & B_{m} \\
\text { not free in the assumptions }
\end{array}
$$

But we are supposing that $x$ is free in $\Gamma$, so that we can replace $S_{I}$ by $R_{I}$ in $\varpi$ to get a proof of $A$ from $\Gamma$, proofs $\pi_{i}$ and the introduction rule for $R$.

\section{THEOREM 9.}

A proof of the sequent $\Gamma \vdash C$ in $\mathrm{LB}^{+}$using only identity and super rules can be transformed into a proof in $\mathrm{NJ}^{+}$of $C$ from $\Gamma$ using only super rules.

Proof. We prove a stronger result: a proof of the sequent $\Gamma \vdash \Delta$ in $\mathrm{LB}^{+}$using only identity and super rules can be transformed into a proof in $\mathrm{NJ}^{+}$of $C$ from $\Gamma$ using only super rules for some $C \in \Delta$.

By induction on the $\mathrm{LJ}^{+}$proof:

- The last rule is an Axiom rule:

$$
\text { Axiom } \overline{\Gamma, A \vdash A, \Delta}
$$

Then we have a trivial supernatural deduction proof of $A$ from assumption $A$.

- The last rule is a cut rule

$$
\operatorname{Cut} \frac{\Gamma, A \vdash \Delta \quad \Gamma \vdash A, \Delta}{\Gamma \vdash \Delta}
$$

By induction hypothesis on the right subproof there exists a proof $\pi$ of either $A$ or some $C \in \Delta$ from $\Gamma$ using only super rules.

In the second case, $\pi$ is a convenient proof. In the first case, by induction hypothesis on the left subproof, there exists a proof $\varpi$ of some $C \in \Delta$ from $\Gamma$ and $A$ using only super rules. By replacing the assumptions $A$ by $\pi$ in $\varpi$, we obtain a proof of $C$ from $\Gamma$.

- The last rule is a right super rule. We can conclude using Lemma 25 and induction hypothesis.

- The last rule is a left super rule. We can conclude using Lemma 24 and induction hypothesis.

\section{B. Interactions PTS terms and first-order terms}

Lemma 26. The translation is compatible with the $\alpha$ conversion:

$$
|\lambda x: A B|_{\ell}=|\lambda y: A\{y / x\} B|_{\ell}
$$

Proof. By simple induction.

Lemma 27. If $x \notin \ell$,

$$
|A|_{\ell:: x: n i l}\left[\operatorname{lift}\left(\operatorname{cons}\left(|u|_{\ell: n i l}\right)\right)\right] \stackrel{*}{\longleftrightarrow}|\{u / x\} A|_{\ell:: n i l} .
$$

Proof. By induction on A.

- If $A=x$, then $|A|_{\ell: x:: n i l}\left[\operatorname{lift}_{\bar{\ell}}\left(\operatorname{cons}\left(|u|_{\ell:: n i l}\right)\right)\right]=$ $\bar{\ell}+1\left[\operatorname{lift} \overline{\bar{\ell}}_{\overline{\bar{l}}}\left(\operatorname{cons}\left(|u|_{\ell:: n i l}\right)\right)\right]$ and $|\{u / x\} A|_{\ell:: n i l}=$ $|u|_{\ell: n n i l}$. We can conclude with [18, Corollary 3.2].

- If $A=y \in \ell$, then $|A|_{\ell:: x: \text { :nil }}\left[\operatorname{lift}\left(\operatorname{cons}\left(|u|_{\ell:: n i l}\right)\right)\right]=$ $\underline{m}\left[\operatorname{lift} t_{\bar{\ell}}\left(\operatorname{cons}\left(|u|_{\ell:: n i l}\right)\right)\right] \quad$ with $m<\bar{\ell}+1, \quad$ and $|\{u / x\} A|_{\ell: \text { :nil }}=|y|_{\ell:: \text { nil }}=\underline{m}$. We can conclude with [18, Corollary 3.2].

- If $A=y \notin \ell:: x$, then $|A|_{\ell:: x: n i l}\left[\operatorname{lift} t_{\bar{\ell}}\left(\operatorname{cons}\left(|u|_{\ell:: n i l}\right)\right)\right]=$ $y[\text { shift }]^{\bar{\ell}+1}\left[\operatorname{lift}_{\bar{\ell}}\left(\operatorname{cons}\left(|u|_{\ell:: n i l}\right)\right)\right]$, and $|\{u / x\} A|_{\ell: n i l}=$ $|y|_{\ell: n i l}=y[\text { shift }]^{\bar{\ell}}$. By [18, Corollary 5.7],

$$
\begin{aligned}
y[\text { shift }]^{\bar{\ell}+1}\left[\text { lift }\left(\overline{\bar{\ell}}\left(\operatorname{cons}\left(|u|_{\ell: n i l}\right)\right)\right]\right. & \\
& \stackrel{*}{\longleftrightarrow} y[\operatorname{shift}]\left[\operatorname{cons}\left(|u|_{\ell: n i l}\right)\right][\text { shift }]^{\bar{\ell}} \\
& \stackrel{*}{\longleftrightarrow} y[\text { shift }]^{\bar{\ell}}
\end{aligned}
$$


- The interesting case is the abstraction over $x$, with $y \neq$ $x$ :

$$
\begin{aligned}
\mid \lambda x & :\left.A B\right|_{\ell:: x: n i l}\left[\operatorname{lift} t_{\bar{\ell}}\left(\operatorname{cons}\left(|u|_{\ell:: n i l}\right)\right)\right] \\
& =|\lambda y: A\{y / x\} B|_{\ell:: x: \text { nil }}\left[\operatorname{lift}_{\bar{\ell}}\left(\operatorname{cons}\left(|u|_{\ell: n i l}\right)\right)\right] \\
& \stackrel{*}{\longrightarrow} \lambda\left(|\{y / x\} B|_{y: \ell::::: n i l}\left[\operatorname{lift}_{\bar{\ell}+1}\left(\operatorname{cons}\left(|u|_{\ell:: n i l}\right)\right)\right]\right) \\
& \stackrel{*}{\mathrm{IH}} \lambda\left(|\{u / x\}\{y / x\} B|_{y:: \ell: n i l}\right) \\
& =\lambda\left(|\{y / x\} B|_{y: \ell:: n i l}\right) \\
& =|\lambda y: A\{y / x\} B|_{\ell: n i l} \\
& =|\lambda x: A B|_{\ell: n i l} \\
& =|\{u / x\} \lambda x: A B|_{\ell:: n i l}
\end{aligned}
$$

Lemma 11. $|A|_{x: \text { nil }}[\operatorname{cons}(|u|)] \stackrel{*}{\longleftrightarrow}|\{u / x\} A|$.

Proof. Corollary of the previous lemma.

Lemma 12. If $|A| \stackrel{*}{\longrightarrow}$ a then there exists $A^{\prime}$ such that $a \stackrel{*}{\longrightarrow}\left|A^{\prime}\right|$.

Proof. By definition, the $W$-normal form of $a$ is a pure term [18, Definition 3.4], and it can therefore be translated back into some $A^{\prime}$ such that $a \stackrel{*}{\longrightarrow} W\left|A^{\prime}\right|$.

Lemma 28. If $z$ does not appear in $\ell,|A|_{\ell: z: n: n i l}=$ $\{\underline{1} / z[$ shift $]\}\left(|A|_{\ell:: n i l}\left[\right.\right.$ lift $t_{\bar{\ell}}($ shift $\left.\left.)\right]\right)$

Proof. By induction on $A$.

- If $A=z$, then $|A|_{\ell:: z: n i l}=\underline{\bar{\ell}+1}$ and $\{\underline{1} / z[$ shift $]\}\left(|A|_{\ell:: \text { il }}\left[\right.\right.$ lift $t_{\bar{\ell}}($ shift $\left.\left.)\right]\right) \quad-\quad=$ $\{\underline{1} / z[$ shift $]\}\left(z[\text { shift }]^{\bar{\ell}}\left[\right.\right.$ lift $t_{\bar{\ell}}($ shift $\left.\left.)\right]\right) \quad$ which is by [18, Corollary 5.5] $\{\underline{1} / z[$ shift $]\}\left(z[\text { shift }]^{\bar{\ell}+1}\right)=$ $\underline{1}[\text { shift }]^{\bar{\ell}}$ which is by definition of the scheme $\underline{\bar{\ell}+1}$.

- If $A=y \in \ell$, then $|A|_{\ell:: z: n i l}=\underline{m}$ with $m<\bar{\ell}$ and $\quad\{\underline{1} / z[$ shift $]\}\left(|A|_{\ell:: n i l}[\right.$ lift $\overline{\bar{\ell}}($ shift $\left.)]\right)=$ $\{\underline{1} / z[$ shift $]\}\left(\underline{m}\left[\right.\right.$ lift $t_{\bar{\ell}}($ shift $\left.\left.)\right]\right)$ which is by $[18$, Corollary 3.2] $\{\underline{1} / z[$ shift $]\} \underline{m}=\underline{m}$.

- If $A=y \notin \ell:: x$, then $|A|_{\ell:: z:: n i l}=y[\text { shift }]^{\bar{\ell}+1}$ and $\quad\{\underline{1} / z[$ shift $]\}\left(|A|_{\ell:: n i l}\left[\right.\right.$ lift $t_{\bar{\ell}}($ shift $\left.\left.)\right]\right)=$ $\{\underline{1} / z[$ shift $]\}\left(y[\text { shift }]^{\bar{\ell}}\left[\right.\right.$ lift $t_{\bar{\ell}}($ shift $\left.\left.)\right]\right)$ which is by [18, Corollary 5.5] $\{\underline{1} / z[$ shift $]\}\left(y[\text { shift }]^{\bar{\ell}+1}\right)=$ $y[\text { shift }]^{\bar{\ell}+1}$.
- The interesting case is the abstraction over $z$, with $y \neq$ $z$ :

$$
\begin{aligned}
& |\lambda z: A B|_{\ell: z:: n i l} \\
& ={ }_{\alpha} \quad|\lambda y: A\{y / z\} B|_{\ell: z:: n i l} \\
& =\lambda\left(|\{y / z\} B|_{y: \ell:: z:: n i l}\right) \\
& \stackrel{*}{\mathrm{IH}} \lambda\left(\{\underline{1} / z[\text { shift }]\}\left(|\{y / z\} B|_{y: \ell:: n i l}\left[\text { lift }_{\bar{\ell}_{+1}}(\text { shift })\right]\right)\right) \\
& =\{\underline{1} / z[\text { shift }]\} \lambda\left(|\{y / z\} B|_{y: \ell:: n i l}\left[\text { lift } t_{\bar{\ell}+1}(\text { shift })\right]\right) \\
& \longleftarrow \quad\{1 / z[\text { shift }]\}\left(\lambda|\{y / z\} B|_{y:: \ell: n i l}\right)\left[\text { lift } t_{\bar{\ell}}(\text { shift })\right] \\
& =\{\underline{1} / z[\text { shift }]\}\left(|\lambda y: A\{y / z\} B|_{\ell:: n i l}\right)\left[\text { lift } t_{\bar{\ell}}(\text { shift })\right] \\
& =\alpha \quad\{\underline{1} / z[\text { shift }]\}\left(|\lambda z: A B|_{\ell: n i l}\right)\left[\text { lift } t_{\bar{\ell}}(\text { shift })\right]
\end{aligned}
$$

Lemma 13. $|A|_{z: n i l}=\{\underline{1} / z[$ shift $]\}|A|_{\text {nil }}[$ shift $]$

Proof. Corollary of the previous lemma

Lemma 14 (Reduction under $\Pi$ ). - If $\left\langle s_{1}, s_{2}, s_{3}\right\rangle \in \mathcal{R}$, $\Gamma \vdash_{\mathrm{PTS}} A: s_{1}$ and $\Gamma[x: A] \vdash_{\mathrm{PTS}} B: s_{2}, \quad x$ is not free in $b, a \stackrel{*}{\longrightarrow}|A|^{\Gamma}$ and $b[\operatorname{cons}(x)] \longrightarrow|B|^{\Gamma[x: A]}$ then $\dot{\pi}_{\left\langle s_{1}, s_{2}, s_{3}\right\rangle}(a, b) \stackrel{*}{\longleftrightarrow}|\Pi x: A B|^{\Gamma}$.

- If $\dot{\pi}_{\left\langle s_{1}, s_{2}, s_{3}\right\rangle}(a, b) \stackrel{*}{\longrightarrow}|C|$ then there exists $A, B$ such that $C=\Pi x: A B$.

Proof.

$|\Pi x: A B|$

$$
\begin{aligned}
& =\quad \dot{\pi}_{s}\left(a,|B|_{x:: n i l}\right) \\
& \underset{\text { Lem. 13 }}{\stackrel{*}{\longrightarrow}} \quad \dot{\pi}_{s}\left(a,\left(\{\underline{1} / x[\text { shift }]\}|B|_{\text {nil }}[\text { shift }]\right)\right) \\
& \stackrel{*}{\stackrel{*}{L e m .13}} \quad \dot{\pi}_{s}(a,(\{\underline{1} / x[\text { shift }]\} b[\operatorname{cons}(x)][\text { shift }])) \\
& {[18, \stackrel{*}{\text { Cor. }} 5.9] \quad \dot{\pi}_{s}(a,(\{\underline{1} / x[\text { shift }]\} b[\text { lift }(\text { shift })][\operatorname{cons}(x[\operatorname{shift}])]))} \\
& \underset{x \text { not free in } b}{=} \quad \dot{\pi}_{s}(a,(b[\text { lift }(\text { shift })][\operatorname{cons}(\underline{1})])) \\
& \underset{[18, \text { Lemma }}{\stackrel{*}{\rightleftarrows}]} \dot{\pi}_{s}(a, b)
\end{aligned}
$$

\section{Why are several $\dot{\pi}$ necessary?}

Because of rule $(2)_{E 1}$ : in the calculus of construction, we could get Girard's paradox:
$(2)_{I}^{\langle\square, *, *\rangle}$
(2) ${ }_{E 1}^{\langle *, * * *} \frac{F^{+} \varepsilon(\dot{\pi}(\star, \underline{1}), \star)}{F^{+} \varepsilon(\star, \star)}$ 